\title{
Localization of Damages in Plain And Riveted Aluminium Specimens using Lamb Waves
}

\author{
Yogesh S. Andhale \\ Department of Mechanical Engineering, D.Y Patil School of Engineering Academy, Ambi, Pune, India.
}

\begin{abstract}
Faeez. A. Masurkar
Smart Engineering Asset Management Laboratory (SEAM) and Croucher Optical Non destructive Testing and Quality Inspection Laboratory (CNDT), Department of Systems Engineering and Engineering Management, City University of Hong Kong, Hong Kong.
\end{abstract}

Nitesh. P. Yelve
Department of Mechanical Engineering, Fr. C. Rodrigues Institute of Technology, Vashi, Navi Mumbai, India.

(Received 15 February 2018; accepted 20 June 2018)

The Lamb wave-based localization of damage is presented here separately for the plain and riveted aluminium ( $\mathrm{Al}$ ) specimens. The first part of this paper deals with the experimental damage localization of the plain Al specimen using Lamb waves and four piezoelectric wafer (PW) transducers. The PW transducers mounted onto the specimen in a collocated way are used to actuate and sense Lamb waves. The responses are obtained for both the pristine and damaged states of the $\mathrm{Al}$ specimen. The signal processing is carried out on the residual response using the continuous wavelet transform (CWT), and time of arrival (TOA) data is obtained for each collocated actuatorsensor pair. The TOA data of the wave reflected from the damage is used in the two arrival time difference and astroid algorithms to locate the damage in an enclosed area. The genetic optimization (GO) method is used to further refine the location of damage within the enclosed area obtained using astroid algorithm. The second part of the paper deals with the localization of a faulty rivet in a riveted specimen. The responses are obtained in the cases of both healthy and faulty riveted specimens. The presence of a faulty rivet is indicated by the inflation in amplitude of the second harmonic. A new algorithm is therefore proposed by the authors to localize the faulty rivet, using the spectral content information. The results obtained through both the studies manifest the ability of the proposed methods for locating different types of defects and faulty rivets using an array of PW transducers.

\section{INTRODUCTION}

Structural health monitoring (SHM) refers to a system having the ability to anticipate and interpret the changes in a structure in order to improve the reliability of the system and thereby reducing the overall life-cycle costs. SHM of aircraft structures is a flourishing field and of paramount importance, as their failure may lead to loss in the economy, and most importantly, the loss of life. An aircraft usually operates in very savage conditions and because of this, there is a need to implement different types of non-destructive tests and evaluations. There are several inspection methods currently being applied to appraise the health of structures in service namely visual inspections, penetrant liquids, magnetic particle-based inspections, Eddy currents, radiography, and ultrasonic testing. ${ }^{1-3}$ Most of these techniques are time consuming, costly, and need the structures under inspection to be in downtime. Among these, ultrasonic testing using Lamb waves has been studied for several years by many researchers. The Lamb wave-based testing offers many advantages, such as large inspection areas because of long distance propagation with lesser attenuation, no need of dismantling and direct access to the structures, and adjustable frequency range for investigation of different sizes of damages. ${ }^{2,3}$ A damage detection system based on ultrasonic Lamb waves requires transducers for the actuating and sensing of elastic waves to interrogate the structures. The vital issues of concern for this system are the multimodal and dispersive nature of Lamb waves and the behaviour of the transducers in action. Due to this, various types of transducers have been invented, out of which piezoelectric wafer (PW) transducers have mustered much attention as they are cost-effective, small, and can be easily bonded onto the structure. ${ }^{4}$ Lemistre et al. successfully detected the structural damage using Lamb waves generated by piezoelectric transducers. ${ }^{5}$

A lot of research is going on around the world for damage detection in metallic and composite plates using Lamb waves for past few decades. Raghavan and Cesnik presented the experimental and analytical survey of candidate methods used for damage detection in thin plate structures. ${ }^{1}$ Giurgiutiu et al. gave a brief review of the Lamb wave principles with emphasis on understanding of the particle motion and group velocity dispersion. ${ }^{2} \mathrm{Su}$ et al. gave a comprehensive review on the state of the art of Lamb wave-based damage identification approaches for composite structures, addressing the advances and achievements in these techniques in the past decades, the unique characteristics and mechanisms of Lamb waves in laminated composites, approaches in wave mode selection, generation and collection, modelling and numerical simulation techniques, signal processing and identification algorithms, and sensor network technology for practical utility. ${ }^{3}$

Masurkar et al. presented an experimental and numerical 
study using the finite element method for single and multi-site damage localization in a thin $\mathrm{Al}$ and a woven fabric composite laminate. ${ }^{6}$ A similar study is also carried out by the same authors, wherein, the Lagrange optimization method is used to refine the location of damage within an enclosed area as defined by the algorithm. ${ }^{7}$ Gangadharan et al. carried out the experiments on both healthy and damaged $\mathrm{Al}$ and composite plates. ${ }^{8}$ Continuous wavelet transform (CWT) is used to extract the time of arrival (TOA) data from the residual response. This TOA data is used in the geodesic algorithm to get the damage location. Hamstad et al. examined the application of CWT to improve the accuracy of acoustic emission (AE) source location. ${ }^{9}$ They examined the cases of both large plate without edge reflections and small plate with multiple edge reflection. Ambrozinski et al. developed LISA and EFIT algorithms to model guided wave propagation problems in metallic and composite structures. ${ }^{10}$ The developed procedure helps to simulate wave propagation for the undamaged and damaged structures for different configurations of actuator-sensor locations, shapes of sensor, and frequency range. They implemented the time and frequency domain methods for damage detection. Yingtao et al. presented a damage assessment methodology using a time-frequency signal processing technique for detecting delamination in composite structures with multiple stiffeners. ${ }^{11}$ The delaminations are detected by identifying the converted Lamb wave modes introduced by the structural imperfection and quantified using a signal energy based damage index. The matching pursuit decomposition algorithm is further used to localize the delamination position by solving a set of nonlinear equations. The results show an overall error of around $8 \%$.

$\mathrm{Su}$ and Ye detected delamination in CF/EP composite laminates using fundamental Lamb modes generated and received by distributed piezoelectric transducers. ${ }^{4}$ Wang and Chang reported a practical damage identification approach using distributed piezoelectric transducers. ${ }^{12}$ Ostachowicz et al. used an array of piezoelectric transducers in order to locate damage in thin metallic plates. ${ }^{13}$ They carried out both simulations and experiments in order to verify the effectiveness of the developed algorithms for localizing the damages. Xinglong et al. presented a damage localization algorithm based on the configuration of the PW sensor network. ${ }^{14}$ Time-frequency analysis is used to process the raw signal and extract damage features. Experimental results obtained for the $\mathrm{Al}$ plate with the proposed algorithm gave an error of around $2.41 \%$. Yan et al. proposed a wavelet-based, built-in damage detection and identification algorithm for carbon fibre reinforced polymer (CFRP) laminate. ${ }^{15}$ Combined with the theoretically computed wave velocity, a genetic algorithm (GA) technique is employed to identify the location and size of the damage. The results show an error of around $4.5 \%$.

Khodaei et al. proposed a numerical model for ultrasonic wave propagation in the composite laminates and compared the results with those recorded from the experiments. ${ }^{16}$ A modified delay-and-sum algorithm is then presented for detecting impact damage in the composite plates with and without a stiffener, which is shown to capture and localize damage with only four transducers. However, the error is reported to be around $8.2 \%$. Ostachowicz et al. presented numerical and experimental approaches for damage detection in structures based on the phenomenon of elastic wave propagation. ${ }^{17}$ The results are presented for isotropic and anisotropic plates with damages in the form of fixed mass and notch cuts. Wang et al. presented a technique for detecting fatigue cracks based on a hybrid sensor monitoring system consisting of a combination of intelligent coating monitoring (ICM) and piezoelectric sensors. ${ }^{18}$ A probability of detection (POD) model that quantifies the reliability of damage detection for a sensor is used to evaluate the weight factor for the ICM and piezoelectric sensors. Masurkar et al. presented a damage localization approach using a two stage method. ${ }^{19}$ In the first stage, the astroid algorithm is employed to localize the damage as an enclosed area, which is formed as a result of intersection of several curves. These curves generated by the algorithm are then used as a constraint function in the Euclidean and Lagrange optimization methods to further refine the damage location in the enclosed area. However, in the present study, a genetic optimization method is used to optimize the damage location. The optimization problem is formulated in a different manner (see section 6), as compared to Masurkar and Yelve. ${ }^{19}$ Furthermore, the weighted values defined by the correlation coefficient analysis, influences the accuracy in locating the damage.

The damage localization carried out by several authors show that there is a need for the development of a robust method which can predict almost the exact location of damage. This is, indeed, very essential, if the specimen to be investigated has a large surface area. Also, work done related to the localization of faulty rivets in a riveted specimen, is scarce in the literature. This defines the objective of the current research. The present work uses an array of PW transducers bonded onto the surface of an Al plate. The healthy responses are obtained on a pristine Al specimen for each actuator-sensor pair configuration. Damage is created in the same Al plate and the damage state responses are obtained for the same actuator-sensor pair configurations. The developed algorithms are applied to the recorded data and the step-by-step explanation and results are presented in this paper. The results show that the method has promising capabilities to locate the different types of defects with fair accuracy. In the second part of this paper, a new algorithm is developed by the authors to identify and localize the faulty rivet in a riveted specimen. Section 2 of the paper deals with the theory of Lamb waves and in Section 3, the experimental setup used for localizing damage in plain $\mathrm{Al}$ specimens is explained. The implementation of CWT for signal processing is explained in Section 4. The conclusions drawn from the present study are given in Section 10.

\section{THEORY OF LAMB WAVES}

Lamb wave is a type of elastic perturbation propagating in a solid plate with traction-free boundaries on both of its surfaces. ${ }^{1-3,6,7}$ This type of wave phenomenon was first described in theory by Horace Lamb in 1917. Lamb waves propagate in two modes, namely symmetric $(S)$ and anti-symmetric $(A)$. The Rayleigh-Lamb wave equation for the symmetric and antisymmetric modes in an isotropic plate is given by:2,3,6,7,20

$$
\frac{\tan (q h)}{\tan (p h)}+\left\{\frac{4 k^{2} q p}{\left(k^{2}-q^{2}\right)^{2}}\right\}^{ \pm 1}=0
$$

where $p^{2}=\left(\frac{\omega^{2}}{C_{L}{ }^{2}}\right)-k^{2}$ and $q^{2}=\left(\frac{\omega^{2}}{C_{T}{ }^{2}}\right)-k^{2}$. In Eq. (1), + is for symmetric modes, - is for anti-symmetric modes, $h$ is the 


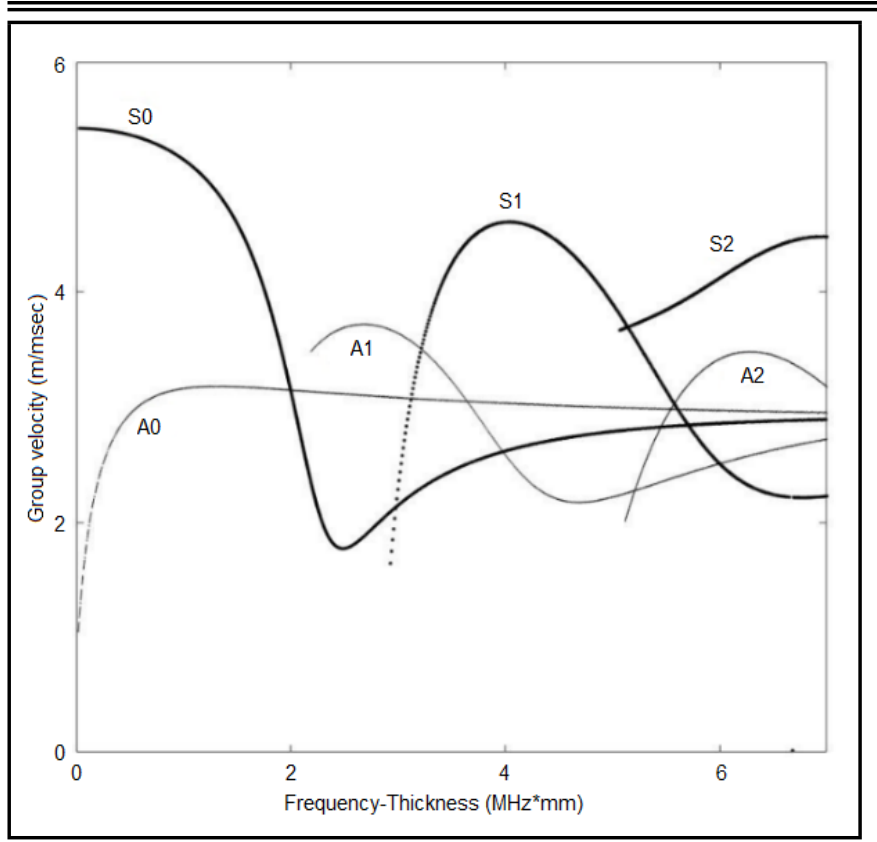

Figure 1. Group velocity dispersion curve (A-Antisymmetric, S-Symmetric modes).

half-plate thickness, $k$ is the wave number, $C_{L}$ is the longitudinal velocity of modes given by $C_{L}^{2}=\frac{\lambda+2 \mu}{\rho}$ and $C_{T}$ is the transverse velocity of the modes given by ${ }_{C_{T}}{ }^{2}=\frac{\mu}{\rho}, k=\frac{\omega}{C_{p}}$, $\lambda=\frac{E v}{(1-2 v)(1+v)}$ and $\mu=\frac{E}{2(1+v)}, \rho$ is the density, $E$ is the Young's modulus, $v$ is Poisson's ratio, $C_{p}$ is the phase velocity, and $\omega$ is the wave circular frequency. In the present study, the group velocity is calculated using the relation:6,7,19,20

$$
C_{g}=C_{p}\left[1-\frac{\omega}{C_{p}} \frac{\partial C_{p}}{\partial \omega}\right]^{-1} .
$$

The Rayleigh-Lamb equations are solved numerically using the bisection algorithm to get the dispersion curves. At lowfrequency thickness products, only two fundamental modes, $S_{0}$ and $A_{0}$ can propagate, as shown in the Fig. 1. The responses are more discernible in this case. However, as the frequencythickness product increases, more number of modes exist and the interpretation of signals becomes more complex. Therefore, one can prefer selective mode generation within a frequency range wherein the effect of dispersion is less. In the present study, the interaction of an $A_{0}$ wave mode with damage is considered. This is due to two reasons. First, the $A_{0}$ wave mode has out-of-plane displacement, which makes it sensitive to surface damage. Secondly, it has a shorter wavelength as compared to $S_{0}$ wave for the same frequency-thickness product, as seen in Fig. 2, and thus, more sensitive to smaller damages.

\section{EXPERIMENTAL SETUP FOR LOCALIZ- ING A DAMAGE IN A PLAIN AL PLATE}

The experimental setup used for localizing a damage in a plain Al plate is shown schematically in Fig. 3. It consists of Tektronix AFG 3021B single channel arbitrary function generator, Tektronix TDS 1002B two channel digital storage oscilloscope, and a personal computer (PC) with LabView software having an online connection with the oscilloscope. The LabView software acts as an interface between the oscilloscope

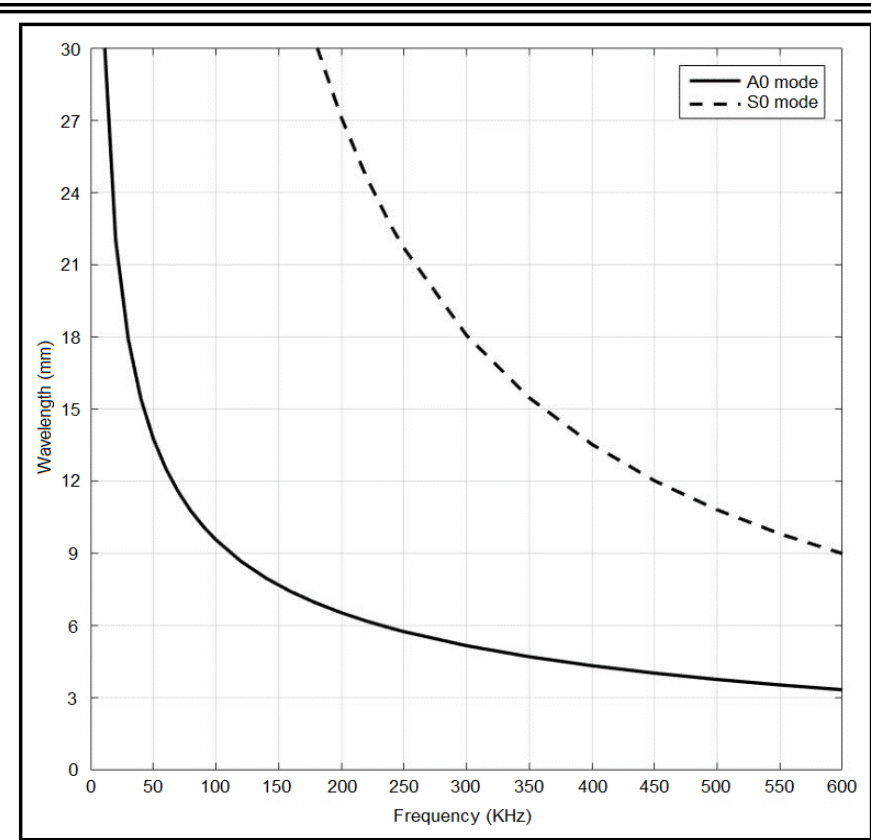

Figure 2. Wavelength dispersion curves for Al plate.

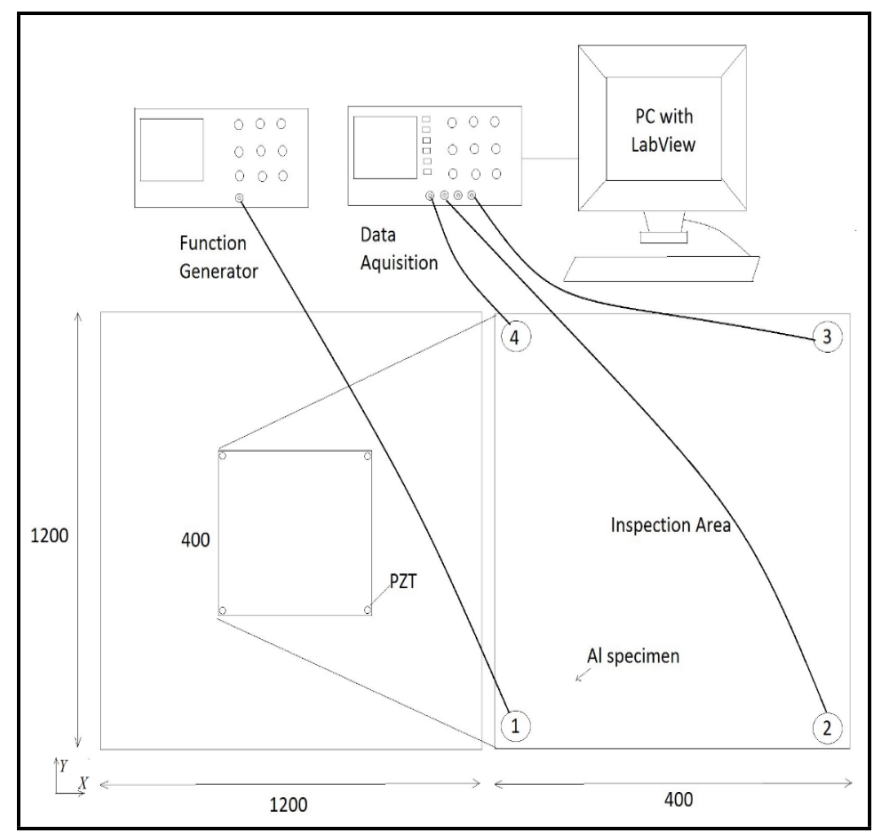

Figure 3. Schematic of the experimental setup.

and PC, and it is used to get the Lamb wave response in a file, which can be imported in MATLAB ${ }^{\odot}$ for offline signal processing. The sampling frequency of the oscilloscope is $5 \mathrm{MHz}$, which is sufficient as per Nyquist criterion. The PW transducers (SP-5H) of $10 \mathrm{~mm}$ diameter and $0.1 \mathrm{~mm}$ thickness are used for actuating and receiving Lamb waves. They are polarized along the thickness direction and bonded onto the plates using a commercially available epoxy based adhesive. The PW circular patches named $T_{1}, T_{2}, T_{3}$, and $T_{4}$ are bonded at locations $(400,400) \mathrm{mm},(800,400) \mathrm{mm},(800,800) \mathrm{mm}$, and $(400,800)$ $\mathrm{mm}$ respectively onto the plate with the planar form dimensions $1200 \mathrm{~mm} \times 1200 \mathrm{~mm}$ as shown schematically in Fig. 3 .

The specimen under study is a $1 \mathrm{~mm}$ thick sheet of Al-5052 alloy with modulus of Elasticity of $70.3 \mathrm{GPa}$, Poisson's ratio of 0.33 , and density of $2680 \frac{\mathrm{kg}}{\mathrm{m}^{3}}$. The excitation tone burst used to excite the Lamb waves is a $10 \mathrm{~V}$ peak-to-peak 8.5 cycle sine 
wave windowed by Gaussian function, and it is supplied with the central frequency of $204 \mathrm{kHz}$. The excitation frequency of $204 \mathrm{kHz}$ is chosen, as per the guidelines given in Giurgiutiu, et al. ${ }^{2}$ The tone burst signal and its frequency spectrum is shown in Fig. 4. Increasing the driving voltage proportionately increases the magnitude of the Lamb waves. Increase in the amplitude also increases the signal to noise ratio, which yields a clearer signal. Higher voltage, however, also tends to increase the drift in the signal, which deteriorates the resolution capabilities of the data acquisition system. The pure sinusoidal shapes appear to excite the Lamb waves most efficiently, since they are periodic, smooth, and have comparatively quick rise time to their peak amplitude, as compared to a parabolic shape. ${ }^{1}$

Gaussian windowing function is used here because it produces a tone burst which has narrow frequency bandwidth with less sidebands, as shown in Fig. 5. The number of cycles considered here for the tone burst is 8.5 because for these many cycles, the Lamb wave modes are seen to be well separated, the frequency bandwidth is less, and the half cycle in the tone burst brings the peak amplitude at the centre, which helps in calculating group velocity. The experiments are carried out at different frequencies on a pristine Al plate and group velocities are calculated. The experimentally obtained group velocities are compared with those obtained numerically and found to be in close agreement, as shown in Fig. 6. This confirms that there is effective actuation and sensing of Lamb waves in the Al plate. The present work uses the astroid algorithm for locating the damage as an enclosed area in the plate. The algorithm uses the time of arrival (TOA) data as the input for forming the enclosed area. The signal processing is carried out here using continuous wavelet transform (CWT) to get the TOA data, which is discussed in the following section.

\section{CONTINUOUS WAVELET TRANSFORM}

According to Daubechies, CWT is defined as: ${ }^{6-9,15,21}$

$$
\Psi(s, p)=\frac{1}{\sqrt{s}} \int_{-\infty}^{\infty} f(t) \varphi^{*}\left(\frac{t-p}{s}\right) d t
$$

where, $f(t)$ is the input signal, $s$ and $p$ are real numbers called scale (or dilation) and position, respectively. The set $\Psi(s, p)$ are the wavelet coefficients of the function $f(t)$ at $(s, p)$, and $\varphi\left(\frac{t-p}{s}\right)$ is the wavelet function while the symbol $*$ denotes the complex conjugate. A CWT allows the division of a continuous time function into wavelets and the construction of a timefrequency representation of a given input signal and is used here to get the TOA data. In the present study, CWT is carried out in MATLAB $^{\odot}$. To make the visualization of the signal discontinuities clear, the Daubechies wavelet ' $\mathrm{db} 4$ ' is selected for the CWT. This is attributed to the fact that the Daubechies wavelet ' $\mathrm{db} 4$ ' is compactly supported, has sharp edges, and it is highly unsymmetrical and due to this, the local disturbances of the analysed signal can be identified. The quantity ' $\mathrm{dbN}$ ' is the short name for the Daubechies wavelet, in which $N$ (here $N=4$ ) is the order of the wavelet. ${ }^{21}$ Figure 7 shows the scaling function and wavelet function of the selected db4. CWT has been used in the literature for processing Lamb wave signals in order to detect damage. ${ }^{5,22}$ Lemistre et al. used CWT to process Lamb wave signals to localize a damage in composite plates. ${ }^{5}$ Ip et al. extracted patch-induced Lamb waves using
CWT. $^{22}$ The two arrival time difference algorithm developed by the authors for damage localization is explained in the following section.

\section{TWO ARRIVAL TIME DIFFERENCE ALGORITHMS}

The collocated PW transducers namely $T_{1}, T_{2}, T_{3}$, and $T_{4}$ are bonded onto the $\mathrm{Al}$ plate as shown in Fig. 3. Consider a case wherein $T_{1}$ acts as an actuator and $T_{2}, T_{3}$, and $T_{4}$ act as sensors. Let $T_{2}$ and $T_{3}$ be located at $\left(T_{2 x}, T_{2 y}\right)$ and $\left(T_{3 x}, T_{3 y}\right)$, respectively, with $V$ being the group velocity of the wave mode in the plate, and $t_{2}$ and $t_{3}$ be the TOA taken by the wave generated from the actuator $T_{1}$ to reach the damage $d$ and further to the sensors $T_{2}$ and $T_{3}$ respectively in the form of reflection from the damage. The difference of TOA taken by the wave to reach these two sensors from the damage is proportional to the distance difference between the damage and sensors. The governing equation can be given by:

$$
\begin{aligned}
& {\left[\overrightarrow{D\left(T_{1} \sim d\right)}+\overrightarrow{D\left(d \sim T_{2}\right)}\right]-} \\
- & {\left[\overrightarrow{D\left(T_{1} \sim d\right)}+\overrightarrow{D\left(d \sim T_{3}\right)}\right]=} \\
= & V_{A_{0}} \times\left(\left(t_{2}-t\right)-\left(t_{3}-t\right)\right)
\end{aligned}
$$

where $d$ refers to the damage and $\overrightarrow{D\left(T_{1} \sim d\right)}$ is the distance between the actuator $T_{1}$ and damage $d, t$ is the time corresponding to the peak of the input pulse. The acronym $V_{A_{0}}$ refers to the group velocity of the $A_{0}$ Lamb wave mode. If damage $d$ has coordinates $\left(d_{x}, d_{y}\right)$ in the planar surface, then the distance between the actuator $T_{1}$ and damage $d$ is given by:

$$
\overrightarrow{D\left(T_{1} \sim d\right)}=\sqrt{\left(T_{1 x}-d_{x}\right)^{2}+\left(T_{1 y}-d_{y}\right)^{2}}
$$

and the distance between the damage $d$ and sensor $T_{2}$ is given by:

$$
\overrightarrow{D\left(d \sim T_{2}\right)}=\sqrt{\left(d_{x}-T_{2 x}\right)^{2}+\left(d_{y}-T_{2 y}\right)^{2}} .
$$

Equation (4) reduces to:

$$
\overrightarrow{D\left(d \sim T_{2}\right)}-\overrightarrow{D\left(d \sim T_{3}\right)}=V_{A_{0}} \times\left(t_{2}-t_{3}\right) .
$$

Thus, the distance difference between the transducers $T_{2}$ and $T_{3}$ from the damage is proportional to the difference between the TOAs. On the similar lines, different equations can be formed for various combinations of actuators and sensors, i.e. $T_{1} T_{2}, T_{1} T_{3}, T_{1} T_{4}, T_{2} T_{1}, T_{2} T_{3}, T_{2} T_{4}, T_{3} T_{1}, T_{3} T_{2}, T_{3} T_{4}, T_{4} T_{1}$, $T_{4} T_{2}$, and $T_{4} T_{3} . T_{1} T_{2}$ combination gives the arrival time $t_{2}$, as the receiving sensor in this case is $T_{2}$. Thus, three different values of $t_{1}, t_{2}, t_{3}$, and $t_{4}$ can be obtained for different collocated actuator-sensor pair combinations.

The above combinations are implemented in the form of equations in MATLAB $^{\odot}$. The area bounded by the PW transducers $(400 \times 400 \mathrm{~mm})$ is meshed using triangular elements in the PDE toolbox of MATLAB ${ }^{\odot}$. This mesh information is then imported as $[p, e, t]$. The array $p$ contains information about the coordinates of nodes, $e$ about the edges, and $t$ about the triangles. The damage coordinates are varied using the $p$ information. The sensor coordinates are fixed as they are bonded onto the plate. The algorithm assumes every node in the mesh as the 


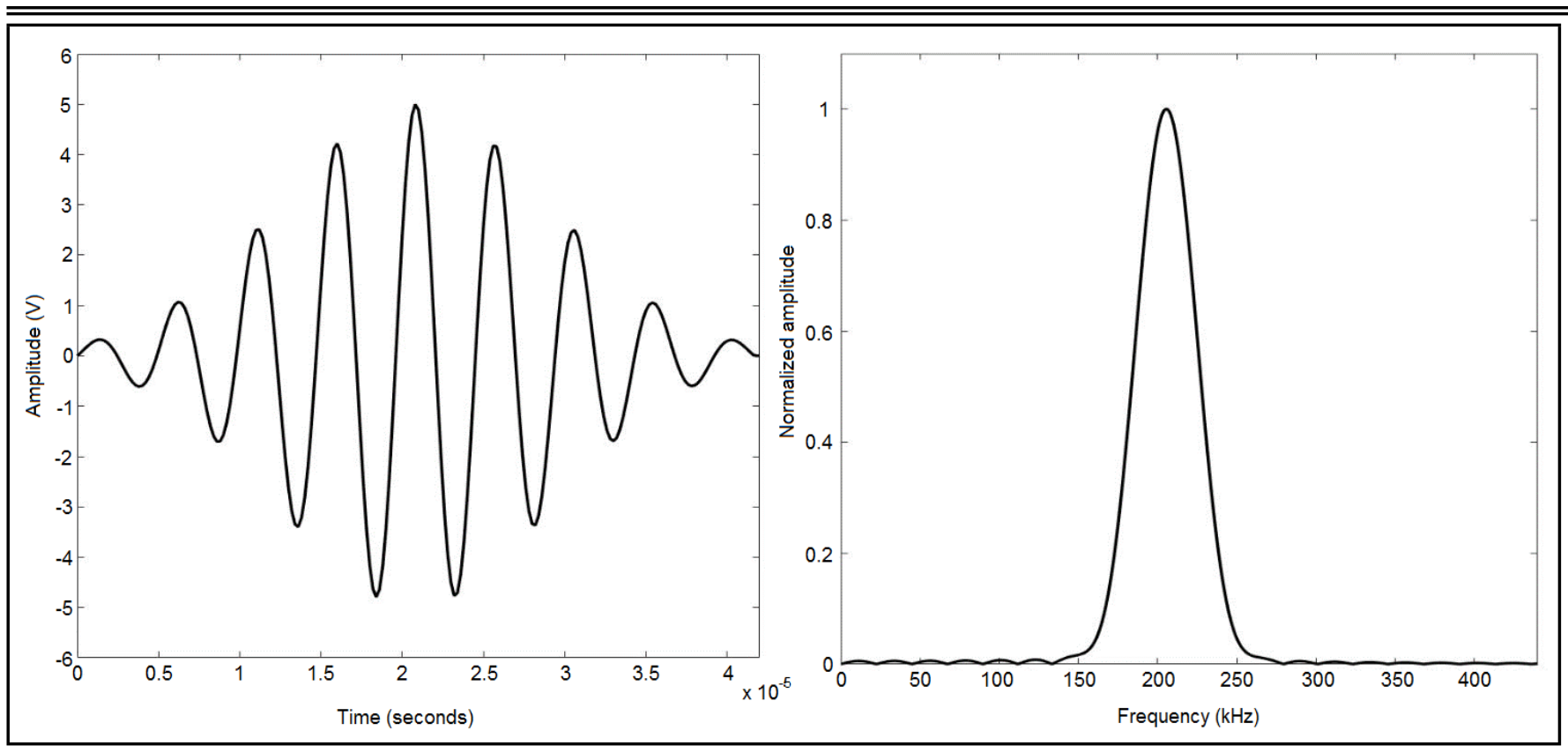

Figure 4. Tone burst signal (left) and its frequency spectrum (right).

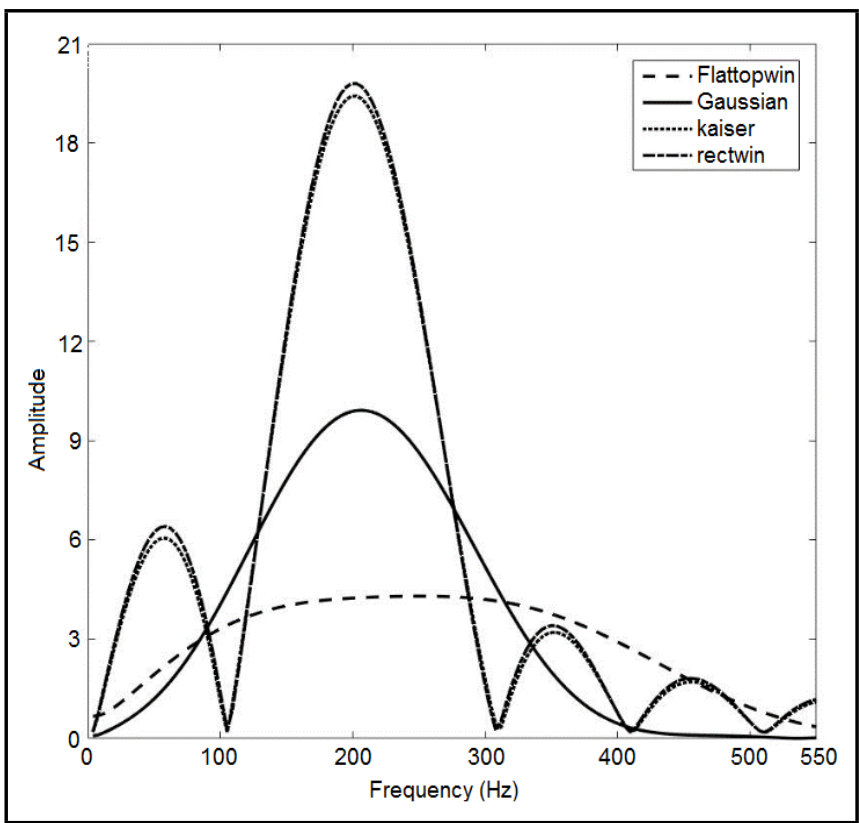

Figure 5. Selection of window.

possible damage location. The algorithm prompts the system to save the information about those nodes where the equation (e.g. Eq. (7)) gets satisfied. This calculation is done for each node in the mesh. Finally, all these nodes are joined together which gives a smooth curve corresponding to the combination of $T_{1} T_{2}$ and $T_{1} T_{3}$ or $\left(t_{2}-t_{3}\right)$, which means $T_{1}$ is acting as an actuator and, $T_{2}$ and $T_{3}$ are receiving the signal $\left(t_{2}-t_{3}\right.$ refers to difference in arrival times). The similar analyses carried out using $T_{1}$ as an actuator and $T_{3}$ and $T_{4}$ as sensors, and subsequently $T_{4}$ and $T_{2}$ as sensors, yield two more curves. The intersection of three curves indicates the damage location which is essentially a small enclosed area. The whole procedure is then repeated with $T_{2}, T_{3}$, and $T_{4}$ as actuators. Thus, in total 12 equations are solved and a refined damage location is identified. The astroid algorithm developed by the authors for damage localization is explained in the following section.

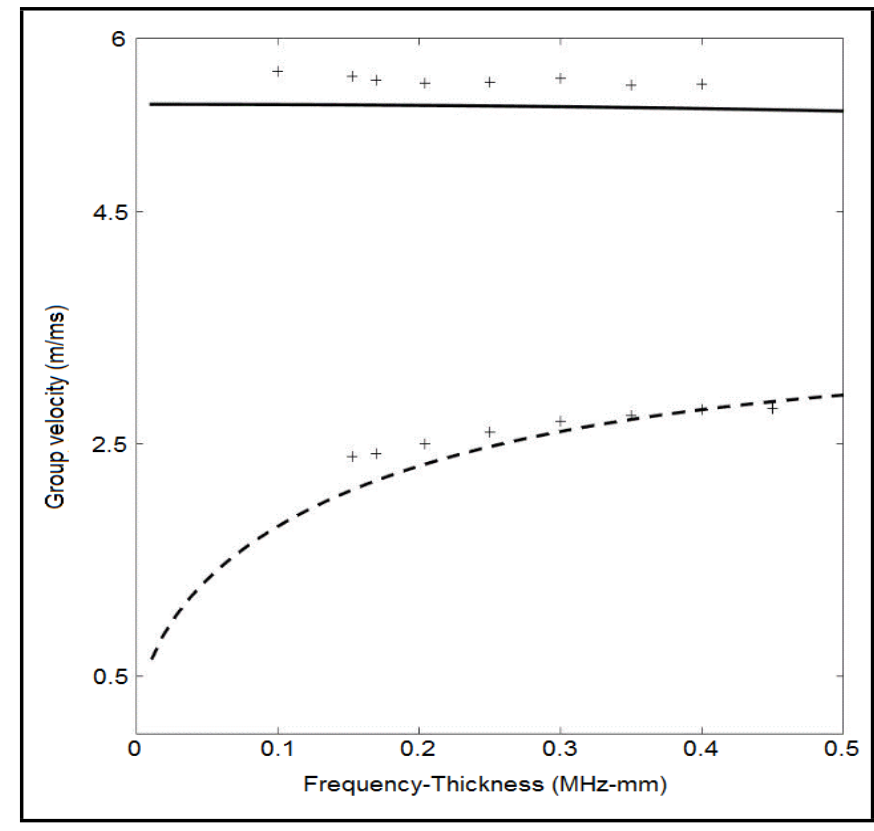

Figure 6. Validation of dispersion curves (+: Experimental results).

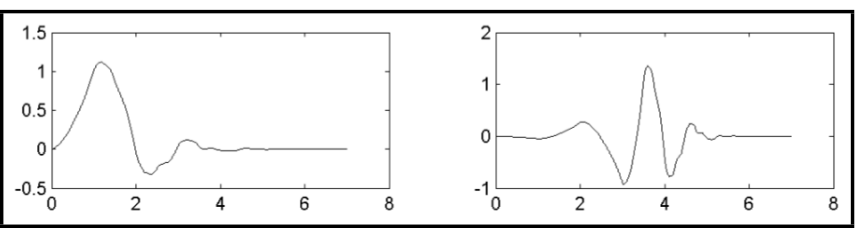

Figure 7. Scaling (left) and wavelet (right) function of db4 wavelet.

\section{ASTROID ALGORITHM}

The astroid algorithm deals with subtracting three arrival times for a particular actuator. The authors' intention is to reduce the number of equations to be solved. In the previous section, one has to solve 12 equations to get the location of the damage, whereas, in this case, only 4 equations need to be solved. Consider a case where $T_{1}$ is acting as an actuator and $T_{2}, T_{3}$ and $T_{4}$ are acting as sensors. The governing equation 


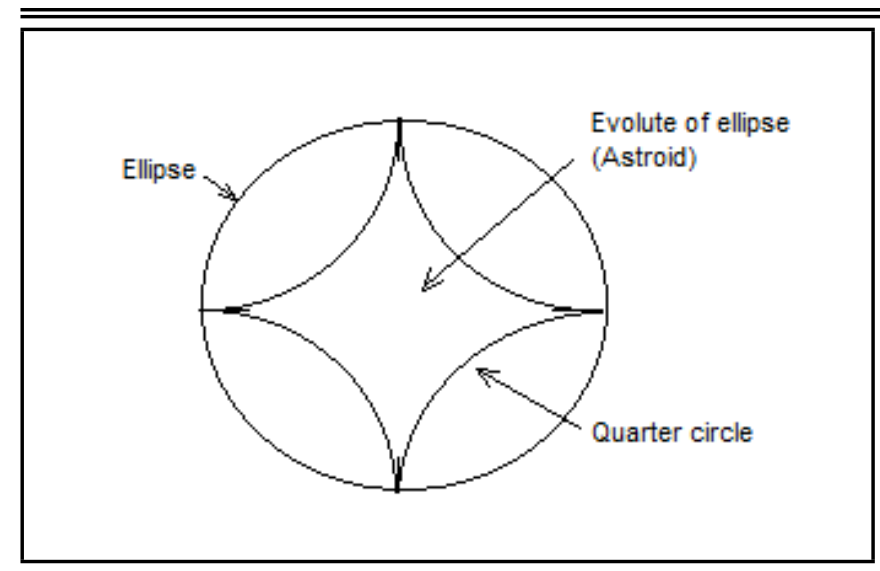

Figure 8. Schematic representation of an astroid.

can be formulated as:

$$
\begin{aligned}
& {\left[\overrightarrow{D\left(T_{1} \sim d\right)}+\overrightarrow{D\left(d \sim T_{2}\right)}\right]-} \\
&-\left[\overrightarrow{D\left(T_{1} \sim d\right)}+\overrightarrow{D\left(d \sim T_{3}\right)}\right]- \\
&-\left[\overrightarrow{D\left(T_{1} \sim d\right)}+\overrightarrow{D\left(d \sim T_{4}\right)}\right]= \\
&=V_{A_{0}} \times\left(\left(t_{2}-t\right)-\left(t_{3}-t\right)-\left(t_{4}-t\right)\right) ;
\end{aligned}
$$

where, $t$ is the input/reference time and $d$ refers to damage. The Eq. (8) can be further written as:

$$
\begin{array}{r}
{\left[\overrightarrow{D\left(d \sim T_{2}\right)}-\overrightarrow{D\left(d \sim T_{3}\right)}-\right.} \\
\left.-\overrightarrow{D\left(d \sim T_{4}\right)}-\overrightarrow{D\left(T_{1} \sim d\right)}\right]= \\
=V_{A_{0}} \times\left(t_{2}-t_{3}-t_{4}+t\right) .
\end{array}
$$

Similarly, three more equations can be formed considering $T_{2}, T_{3}$ and $T_{4}$ as actuators, which give quarter circular arcs with the concerned actuator as its centre. The points of intersection of these quarter circular arcs are joined by an ellipse, as shown schematically in Fig. 8. Figure 8 also gives an idea to the readers about the shape of an astroid. If one draws the evolute of an ellipse, it gives an astroid shape. The enclosed areas obtained in the present study are not perfectly an astroid, but closely resembles to the shape of an astroid. Therefore, this algorithm is termed as the astroid algorithm.

The astroid algorithm generates perfect circles, unlike the curves generated by the two arrival time difference algorithm. The four quarter circles give an enclosed area $(a b c d)$, wherein, the probability of the presence of damage is very high as shown in Fig. 9. The damage location is further refined within this enclosed area using the genetic optimization, which is discussed in following section 7. However, in order to use genetic optimization technique, an objective function needs to be defined. The change in the Lamb wave response for individual sensing paths, in the cases of both healthy and damaged states of plates, are quantitatively measured by the correlation coefficient (CC). The CC of two signals, $x=\left[x_{1}, x_{2}, \ldots, x_{n}\right]$ and $y=\left[y_{1}, y_{2}, \ldots, y_{n}\right]$ is defined as:

$$
\epsilon=\frac{\sum_{i=0}^{n} \sum_{j=0}^{n}\left(x_{i j}-\bar{x}\right)\left(y_{i j}-\bar{y}\right)}{\sqrt{\left(\sum_{i=0}^{n} \sum_{j=0}^{n}\left(x_{i j}-\bar{x}\right)^{2}\right)\left(\sum_{i=0}^{n} \sum_{j=0}^{n}\left(y_{i j}-\bar{y}\right)^{2}\right)}} ;
$$

where $\bar{x}=\frac{1}{n} \sum_{i=1}^{n} x_{i}, \bar{y}=\frac{1}{n} \sum_{i=1}^{n} y_{i}, x$ refers to the healthy state response and $y$ refers to the damaged state response. The value of $\epsilon$ gives the degree of relationship between the two signals, $x$ and $y$. It varies between 0 (when the signals have no linear relationship) and 1 (when each signal is perfectly approximated by the other). Considering a certain actuator-sensor path, the degree of correlation in the responses are calculated. The smaller the value of $\epsilon$, the more closely the damage is located to the considered actuator-sensor path. Conversely, it will approach nearer to unity, if the sensing path is far from the damage. The output of the astroid algorithm is an enclosed area, with four different intersections, as shown by the points $a, b$, $c$, and $d$ in Fig. 9. The authors have formulated an equation which minimizes the sum of squares for $a, b, c$, and $d$ from the damage location. Therefore, the objective function can be formulated as:

$$
\operatorname{Minimize}\left\{\sum_{i=a}^{d}(\overrightarrow{x-x(l)})^{2}+\sum_{j=a}^{d}(\overrightarrow{y-y(\jmath)})^{2}\right\}
$$

where, $i$ and $j$ refer to the intersection points of the enclosed area and $x$ and $y$ refer to the optimal solution. However, from Fig. 9, it is quite evident that the damage location is not symmetrical with respect to the enclosed area. In most of the practical applications, there is a high possibility that the damage is located offset or anywhere within the enclosed area. Thus, in order to generalize the procedure, a weighted function is proposed, which can take into account this shortcoming of Eq. (11). The weights are defined as:

$$
\overleftrightarrow{W_{k, l}}=\frac{1}{\epsilon_{T_{k} T_{l}}}
$$

where, $k$ and $l$ refer to the actuator and sensor numbers. Therefore, the revised objective function becomes:

$$
\begin{aligned}
& \text { F Minimize }\left\{\sum_{i=a}^{d} \overleftrightarrow{W_{k, l}}(\overrightarrow{x-x(l)})^{2}+\right. \\
&\left.+\sum_{j=a}^{d} \overleftrightarrow{W_{k, l}}(\overrightarrow{y-y(J)})^{2}\right\} .
\end{aligned}
$$

In the objective function, the weights $W_{k, l}$ for each intersection are calculated as the inverse of the $C C$, which is surely greater than unity. The weights make the solver realize the position of damage within the enclosed area.

\section{GENETIC ALGORITHM}

The astroid algorithm as explained in the previous section, gives the location of the damage in a plate as an enclosed area. However, the size of the enclosed area may vary from case to case. Thus, a robust technique should be applied, which can further refine the location of the damage in the enclosed area. The genetic algorithm (GA) is best suited for this purpose because of its great flexibility for optimizing nonlinear multi-dimensional problems, its capability to converge to the near optimum of the problem, regardless of the initial guess, and the fact that a GA can be easily parallelized reducing the 


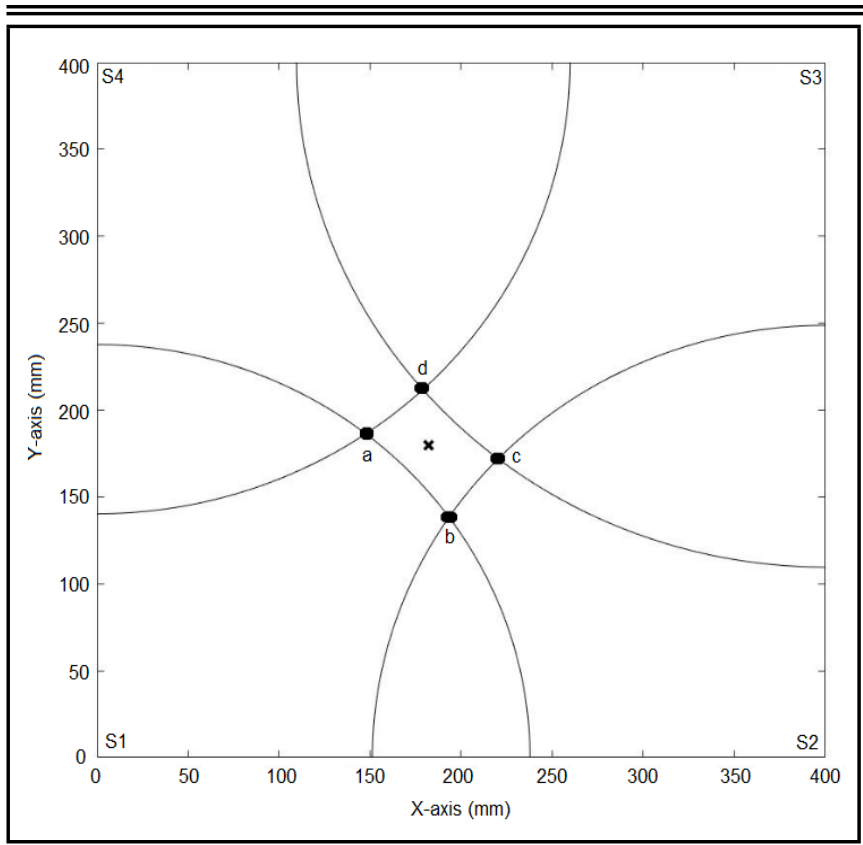

Figure 9. Enclosed area.

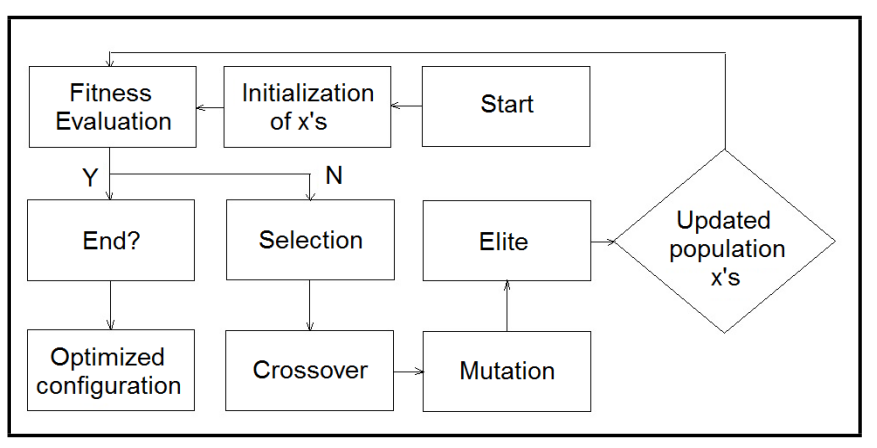

Figure 10. Flowchart of a GA optimization method.

computational effort. Another advantage of the GA is its capability to not to remain trapped in a suboptimal solution.

The GA uses the biological evolution principles, which include the natural selection and survival of the fittest. The parameters, the rules, and switching of the considered problem are represented by a binary combination called the chromosome. The estimated parameters could be integers or floating points, according to the problem. The user selects the number of assigned bits to the parameters by considering the compromise between accuracy and computational time. The goal of the GA is to obtain the optimal 0 s and $1 \mathrm{~s}$ for the chromosome to minimize or maximize the objective function. The user selects the population size, number of children for each set of parents, and probability of mutation, according to the problem, to complete the optimization process quickly and accurately. The flowchart of the GA optimization method is shown in Fig. 10. The genetic program generally starts by randomly assigning $0 \mathrm{~s}$ and $1 \mathrm{~s}$ to the chromosome. The values of the chromosome are updated by a process consisting of five steps, viz. selection of the mating parents, selection of the hereditary chromosome from parents, gene crossover, gene mutation, and creation of the next generation.

\section{DAMAGE SCENARIOS}

The proposed algorithms are tested over three different damage scenarios, viz., a small triangular notch, an extra material added onto the plate (protrusion), and through thickness hole in the plate. The two arrival time difference algorithm explained in Section 5 is implemented in the case of a small triangular notch and the astroid algorithm explained in Section 6 is implemented in the cases of all damage scenarios.

\subsection{Case 1}

A small triangular notch is created in the plate at $(182,180)$ mm with $T_{1}$ at origin, $T_{1}-T_{2}$ as $x$-axis, and $T_{1}-T_{4}$ as $y$-axis as shown in Fig. 11. The depth of the damage along the thickness direction of plate is kept as $0.5 \mathrm{~mm}$.

\subsubsection{Localization of Damage Using the Two Arrival Time Difference Algorithm}

Experiments are carried out at the central frequency of $204 \mathrm{kHz}$ for various combinations of actuator and sensors. The responses obtained for the pristine and damaged plates for $T_{1} T_{2}$ combination are shown in the Figs. 12 and 13, respectively. Damage is created in the same healthy plate after obtaining the pristine state response. This is because the proposed method subtracts the responses obtained at the damaged and pristine states. If one uses another plate, a slight mismatch in the bonding of the PW transducers onto the plates will lead to erroneous residual response. The residual response obtained clearly reveal the presence of additional wave packets due to reflection of $S_{0}$ and $A_{0}$ wave modes from the damage as shown in Fig. 14. The CWT is carried out on the pristine, damaged, and residual responses obtained in the case of $T_{1} T_{2}$ combination as shown in Figs. 15, 16, and 17, respectively. The CWT plot clearly gives the information about the reflection of wave modes coming from the damage.

It is very important to identify the proper peak of the reflected wave packet from the damage. Knowing the exact distance between the actuator and sensor, which is $400 \mathrm{~mm}$, one can easily understand that the peak of the reflected wave packet from the damage cannot give a distance that is less than 400 $\mathrm{mm}$. It should be at least equal to $400 \mathrm{~mm}$. With this logic, the peaks of the wave packets reflected from the damage can be identified and are shown in Fig. 18. Obviously, the second peak corresponds to $A_{0}$, as it propagates with less velocity, as compared to $S_{0}$ mode. The acronym $S_{0} /$ TOA and $A_{0} /$ TOA shown in Fig. 18 refer to the TOA of the $S_{0}$ and $A_{0}$ wave modes reflected from the damage, respectively. However, some additional peaks can also be seen in Fig. 18. These peaks correspond to minor reflections (from other collocated actuators) and noise.

Figure 18 shows that Lamb waves are highly sensitive to the presence of any damage and their propagation is affected by the presence of such discontinuities. These irregularities are revealed by the peaks, as those that can be seen in Fig. 18. The TOA data is obtained from the locations of the peaks. This TOA data is required for both the two arrival time difference and astroid algorithms to locate the damage as an enclosed area. Figs. 19-22 are generated by implementing the two arrival time difference algorithm discussed in Section 5. 


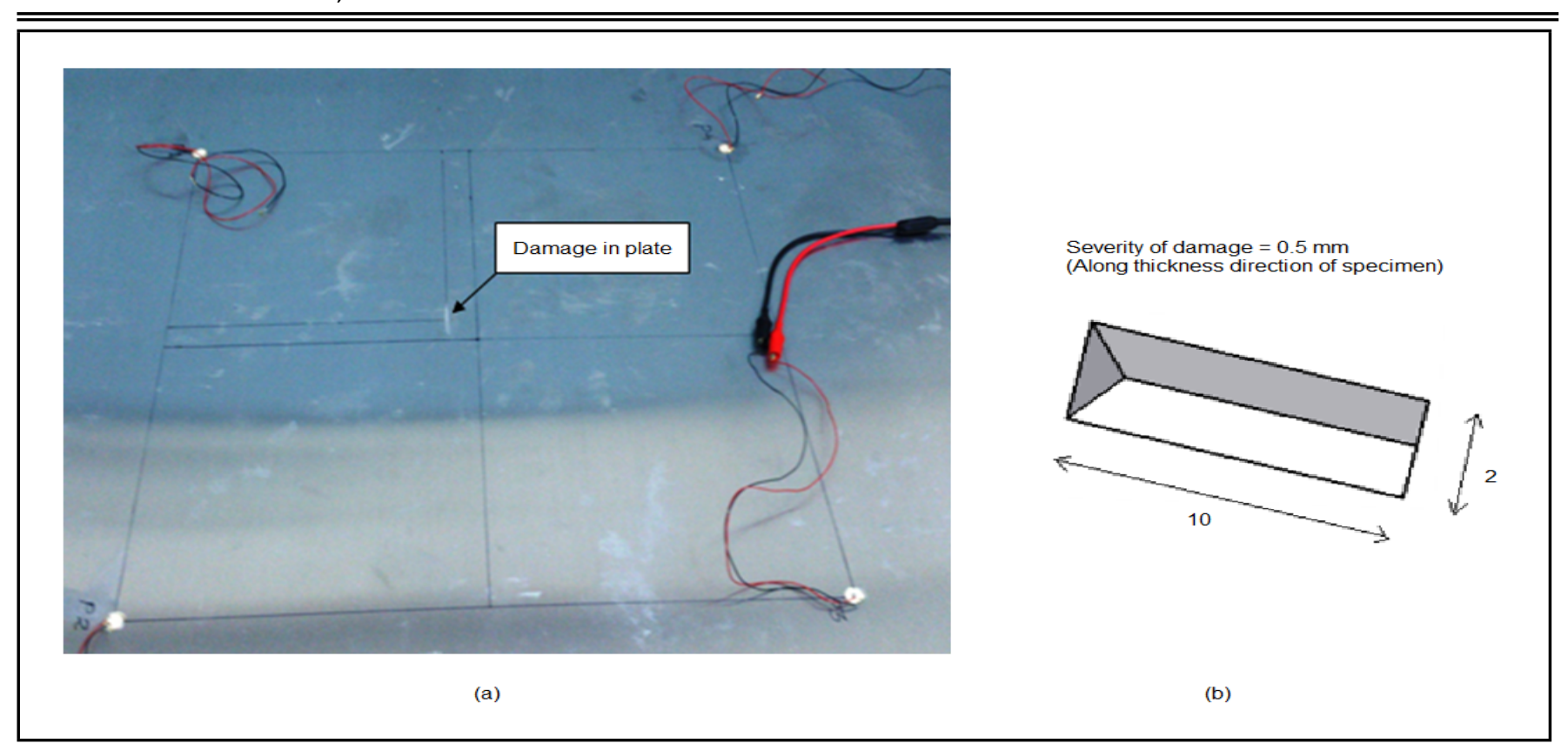

Figure 11. (a) Al plate with the notch (b) Exaggerated view of notch (All dimensions are in $\mathrm{mm}$ ).

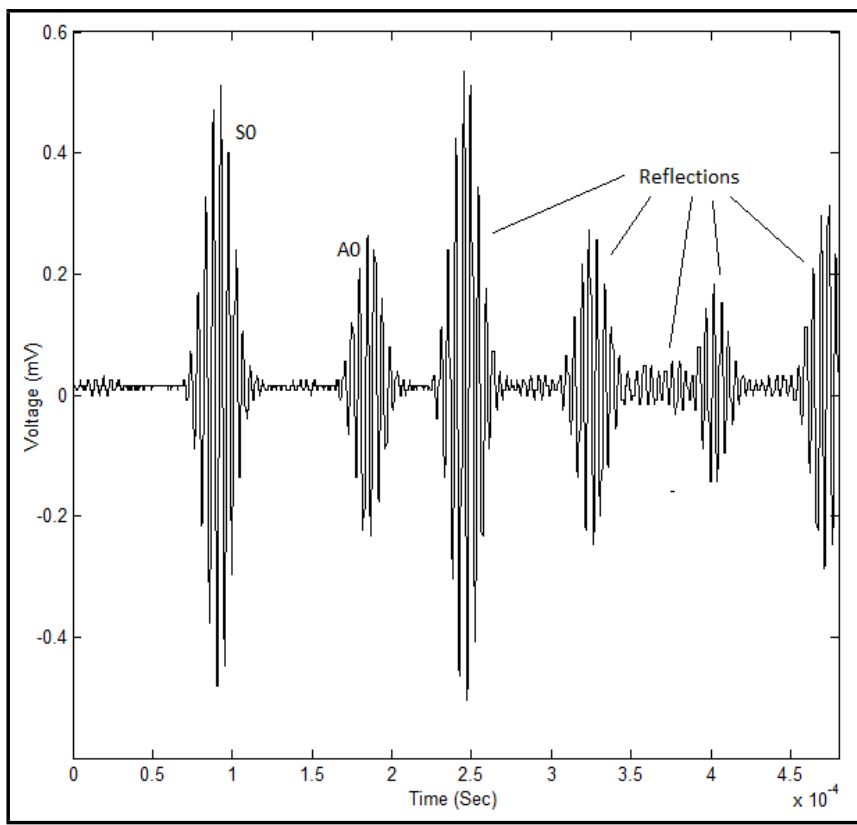

Figure 12. Healthy signal for $T_{1} T_{2}$ combination.

The " $x$ " mark on each figure shows the actual location of damage and the intersection of curves gives the predicted location of damage. As the damage has some finite area, the locations of the damage obtained in each set of experiments differs from the actual one. The results show the location of the damage fairly well in all the cases and the coordinates of the damage obtained in Figs. 19-22 are (142.9, 189.6) mm, (181.3, $134.5) \mathrm{mm},(228.3,187.3) \mathrm{mm}$, and $(209.3,168.5) \mathrm{mm}$, respectively. Due to the significant deviation between the predicted and actual location of defects, an astroid algorithm in combination with GO is developed in the present study to estimate the damage locations precisely and the results are discussed in following subsection.

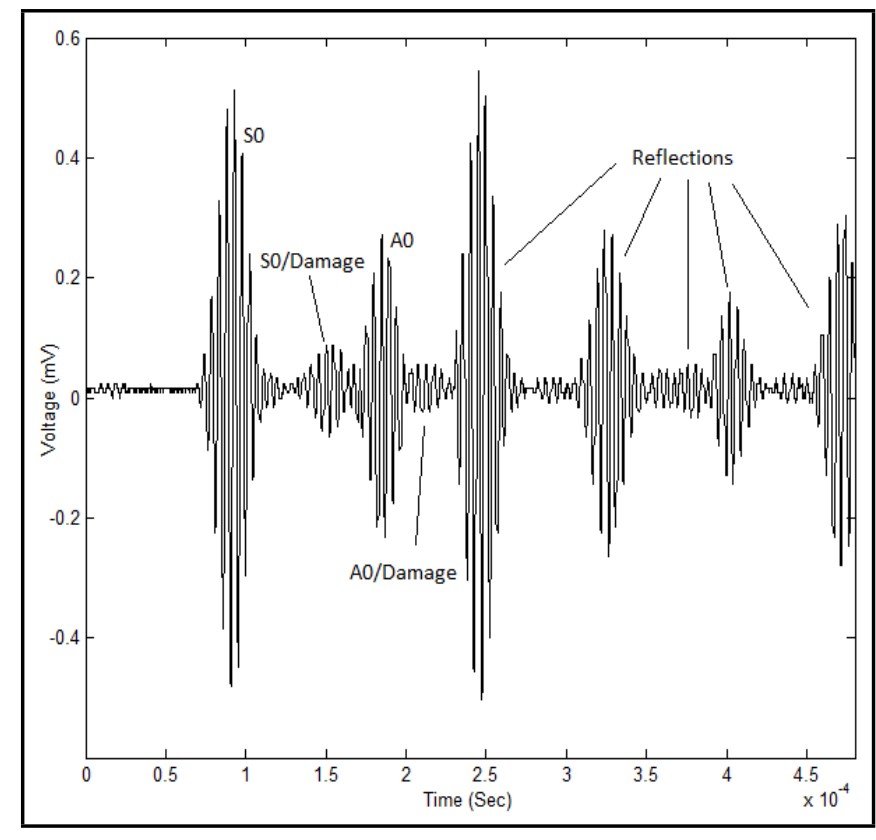

Figure 13. Damaged signal for $T_{1} T_{2}$ combination.

\subsubsection{Localization of Damage Using the Astroid Al- gorithm}

The two-dimensional CC's calculated using Eq. (10) show that $T_{2} T_{4}$ and $T_{3} T_{1}$ are the most affected as can be seen from Fig. 23. It can be justified in two ways: firstly, the damage is located along the diagonals of the plate (which is evident from all actuator-sensor pairs giving less value of $\epsilon$ for diagonal configuration) and secondly, the damage is located closer to the transducer $T_{1}$ and $T_{4}$. The value $\epsilon$ for $T_{3} T_{1}$ is 0.769 , whereas, for $T_{2} T_{4}$, it is 0.777 , as shown in Fig. 23. The result of $\epsilon$ show that the damage is closely located to the transducer $T_{1}$ than $T_{4}$ with respect to enclosed area which agrees quite well with the physical dimensions.

With these settings, the GA runs for 143 generations where convergence takes place. Fig. 24 shows a graphical representation of the evolution of the fitness for the mean and for the 


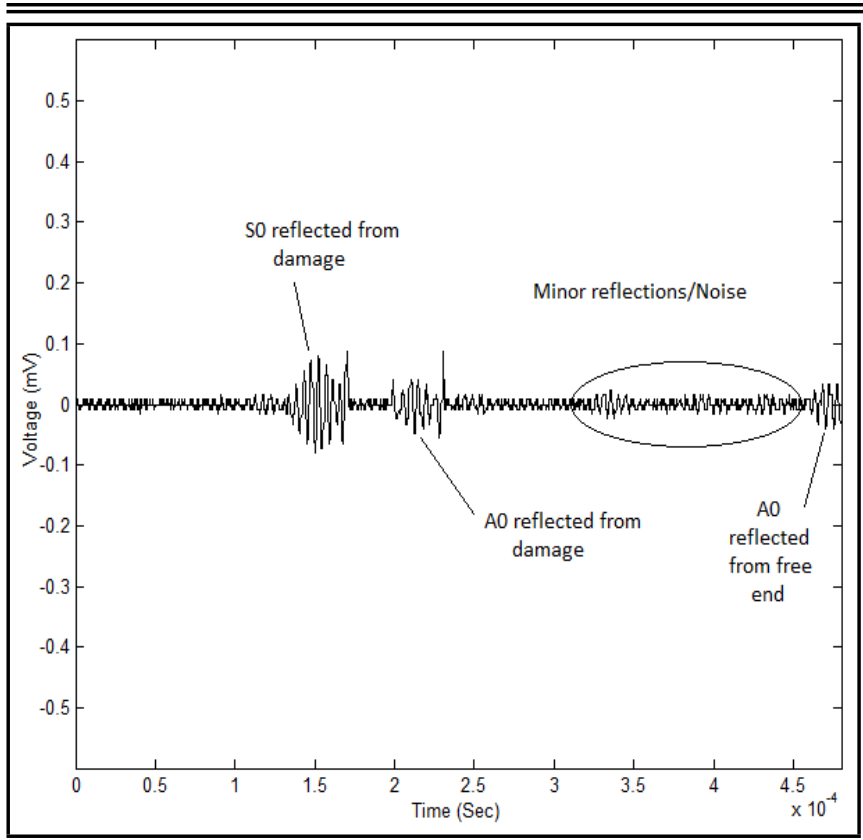

Figure 14. Residual signal for $T_{1} T_{2}$ combination.

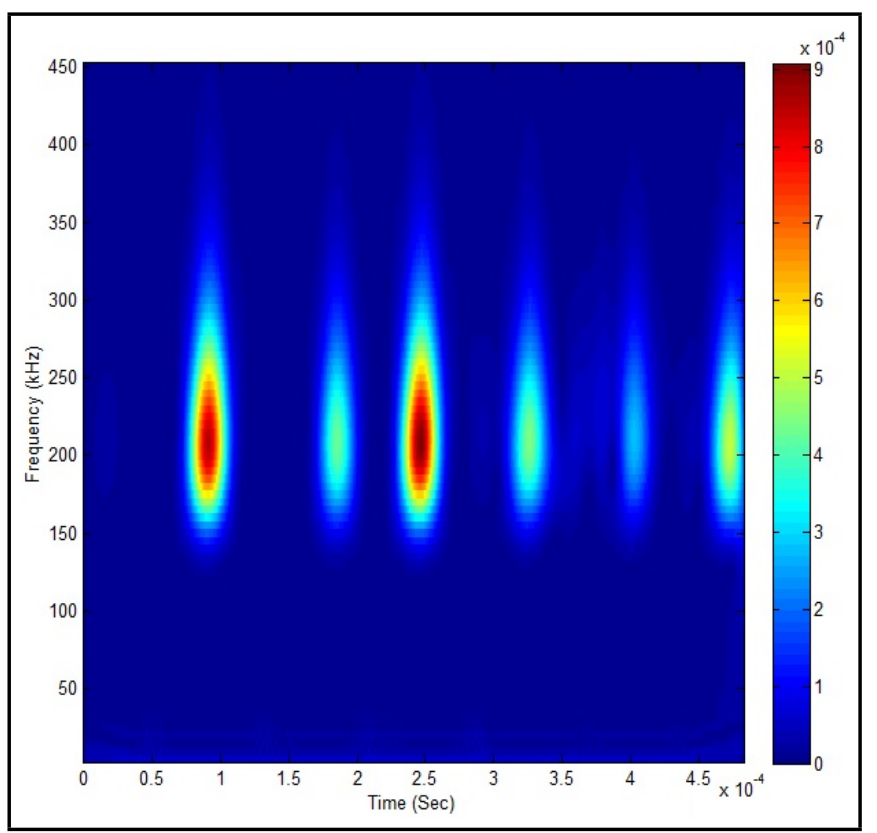

Figure 15. CWT of healthy signal for $T_{1} T_{2}$ combination.

best individual of a population during the inversion procedure. Thus, it can be readily seen that the fitness value is higher at the start of the inversion procedure (Generation 0 ), and converges to a steady state at the end of the inversion procedure (Generation 143). The change in average distance between the individuals over different generations can be seen from Fig. 25. In the present case, it is tried to optimize the location of the defect, wherein, the set of different vectors $X$ (coordinates of damage) corresponds to a complete population in GA, while a single $X$ corresponds to an individual in the population.

The above procedure is executed for each $X$ in the current population. The obtained fitness values for that population are then sorted in a descending order. After this, the current population generates the next population by producing descendants. First, those individuals having superior fitness values are directly recruited in the next generation, according to the princi-

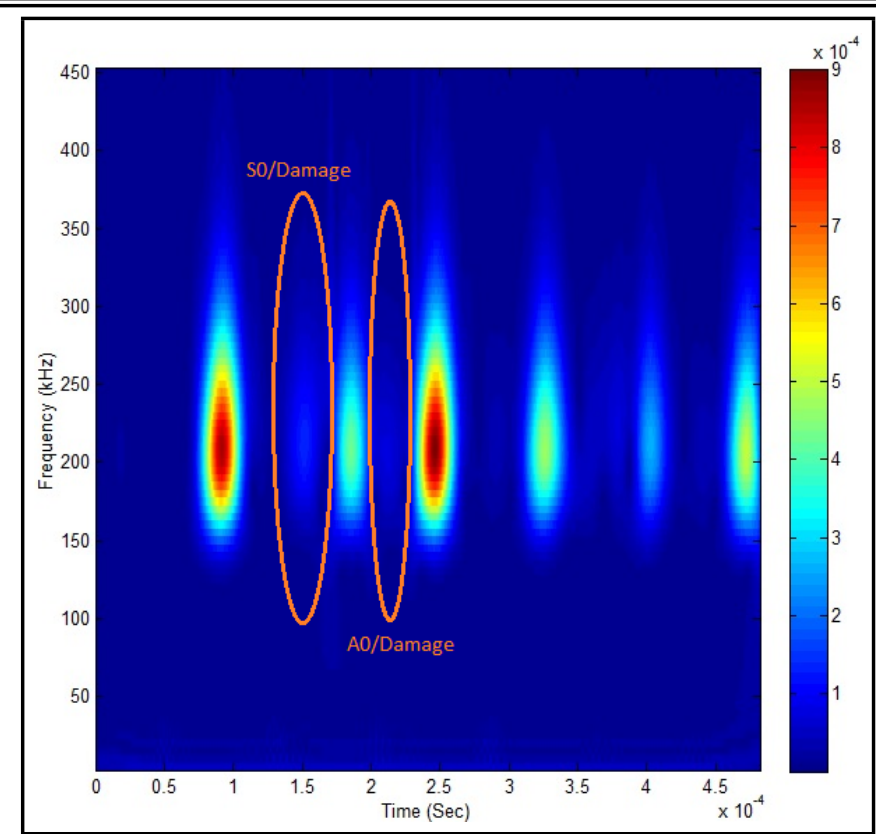

Figure 16. CWT of damaged signal for $T_{1} T_{2}$ combination.

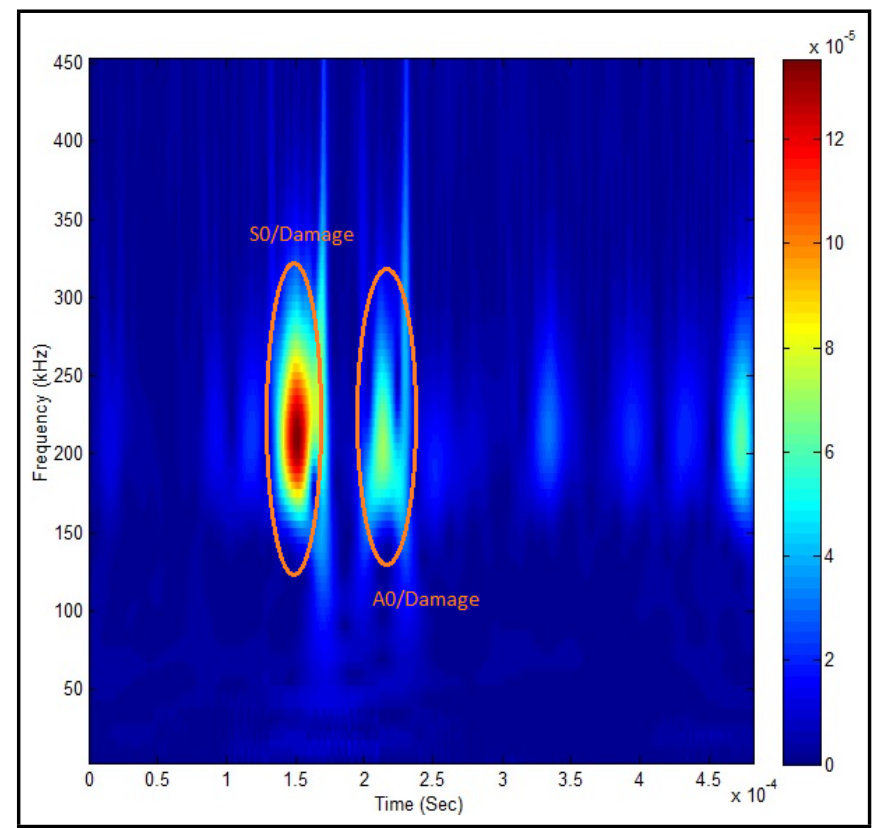

Figure 17. CWT of residual signal for $T_{1} T_{2}$ combination.

ple of elitism. Secondly, crossover children are formed by arbitrarily mixing the characteristics of any two individuals. Lastly, the next population is formed by random mutations of an individual by arbitrarily flipping bit values from 0 to 1 and vice versa, thus resulting in a set of mutated individuals. Once the next generation is created, the procedure is repeated in order to obtain a fitness value of the updated generation. This loop is repeated for many updated populations, until convergence is reached.

The stopping procedure is activated when the variation of the fitness value of both the best individual and the mean of the current population is sufficiently smaller over subsequent generations. Once these conditions are fulfilled, the procedure is stopped and the optimized $X$ is obtained. The GA optimization method gives the coordinates $(182.196,179.135) \mathrm{mm}$ as the damage location which is very close to the actual damage 

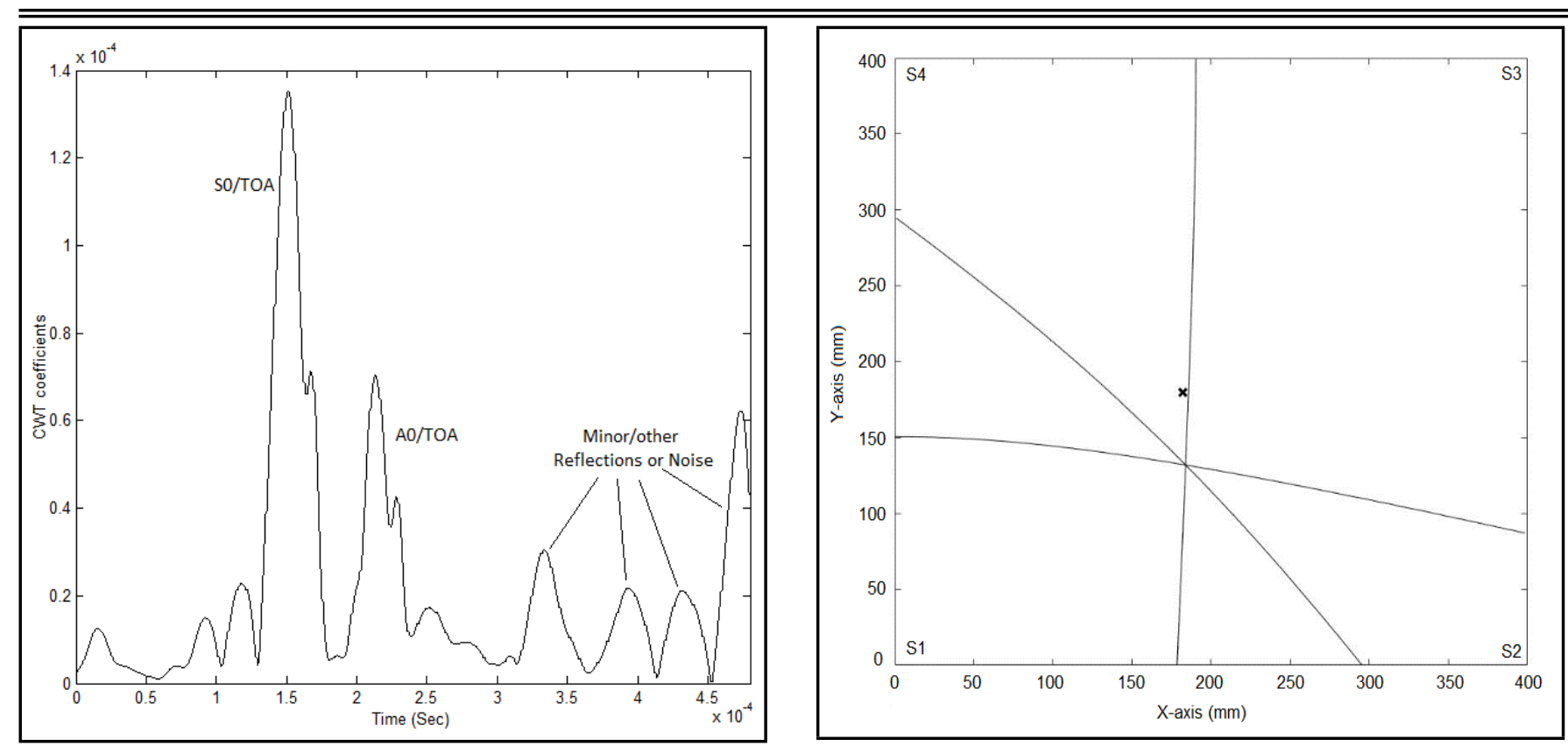

Figure 18. CWT of residual signal (Line plot).

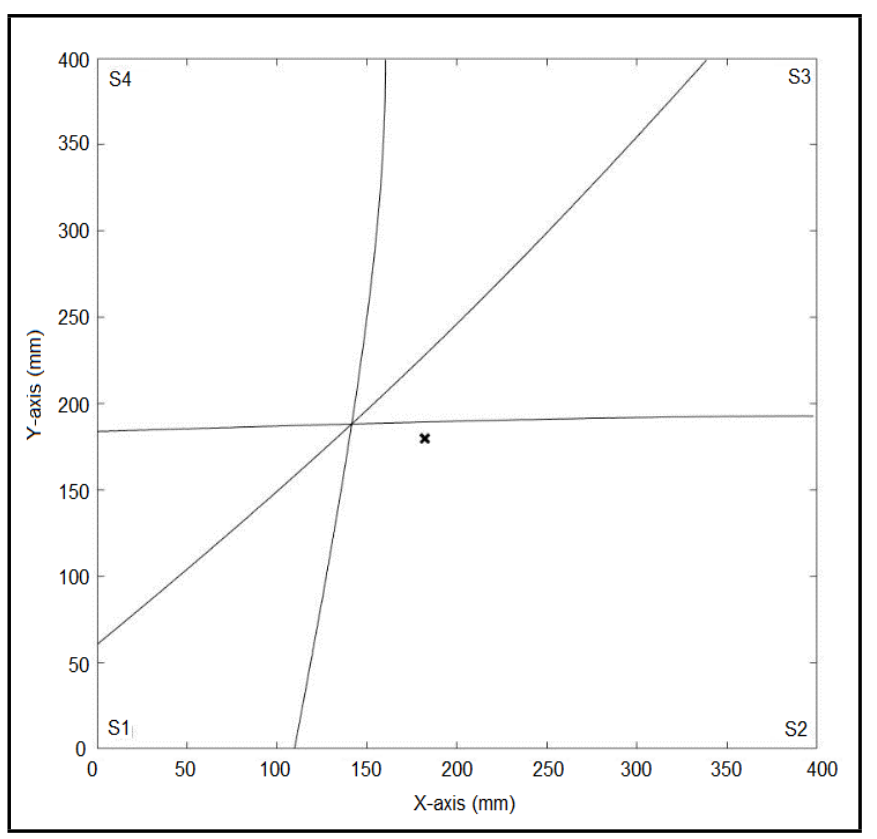

Figure 20. $T_{2}$ acting as an actuator.

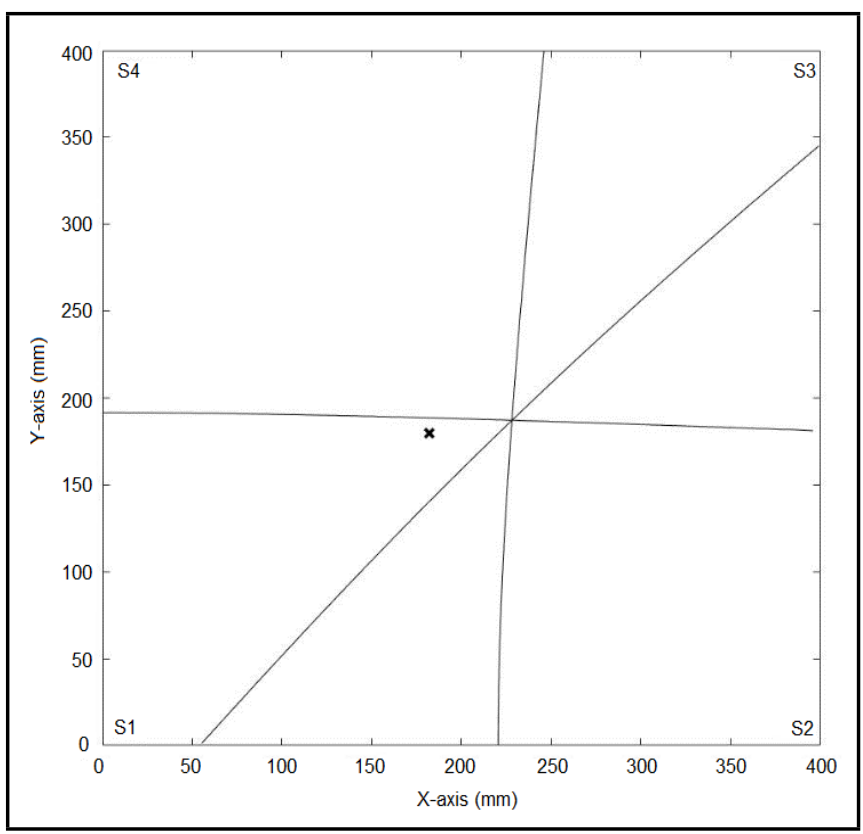

Figure 19. $T_{1}$ acting as an actuator.

Figure 21. $T_{3}$ acting as an actuator.

location $(182,180) \mathrm{mm}$.

\subsection{Case 2}

In this case, an extra material is added onto the plate at the location $(100,100) \mathrm{mm}$. The material has a rectangular planar shape and it is bonded onto the plate using an epoxy-based adhesive, so that it behaves as a protrusion to the plate, as shown in Fig. 26. The experiments are carried out on the plates with and without a protrusion at $204 \mathrm{kHz}$. The responses are recorded for both state and are shown in Figs. 27 and 28, respectively. The response in Fig. 28 shows the reflected wave packet coming from the protusion and captured by transducer $T_{2}$. The GO method predicted the location of protrusion as $(99.056,100.719) \mathrm{mm}$, which is very close to the actual damage location of $(100,100) \mathrm{mm}$. For brevity, the optimization results are not shown here.

\subsection{Case 3}

In this case, a through thickness hole is investigated as shown in the Fig. 29. The hole of $0.5 \mathrm{~mm}$ diameter is created at (300, 100) $\mathrm{mm}$. The responses are obtained at $204 \mathrm{kHz}$. The damage state response clearly indicates the reflected wave packet due to the hole, as shown in Fig. 30. The signal processing is carried out using CWT for each of the residual response obtained in the Cases 2 and 3. The TOA data is extracted in each case and is used independently in the astroid algorithm. The algorithm generates astroidal shapes in both the cases, which show the damage location as an enclosed area. For brevity, the algorithm outputs are not shown for the Cases 2 and 3. The GO method predicts the location of the hole as $(300.948,101.851)$ $\mathrm{mm}$, which is very close to the actual damage location of (300, 100) $\mathrm{mm}$. For brevity, the optimization results are not shown here. The second part of the study deals with the localization 


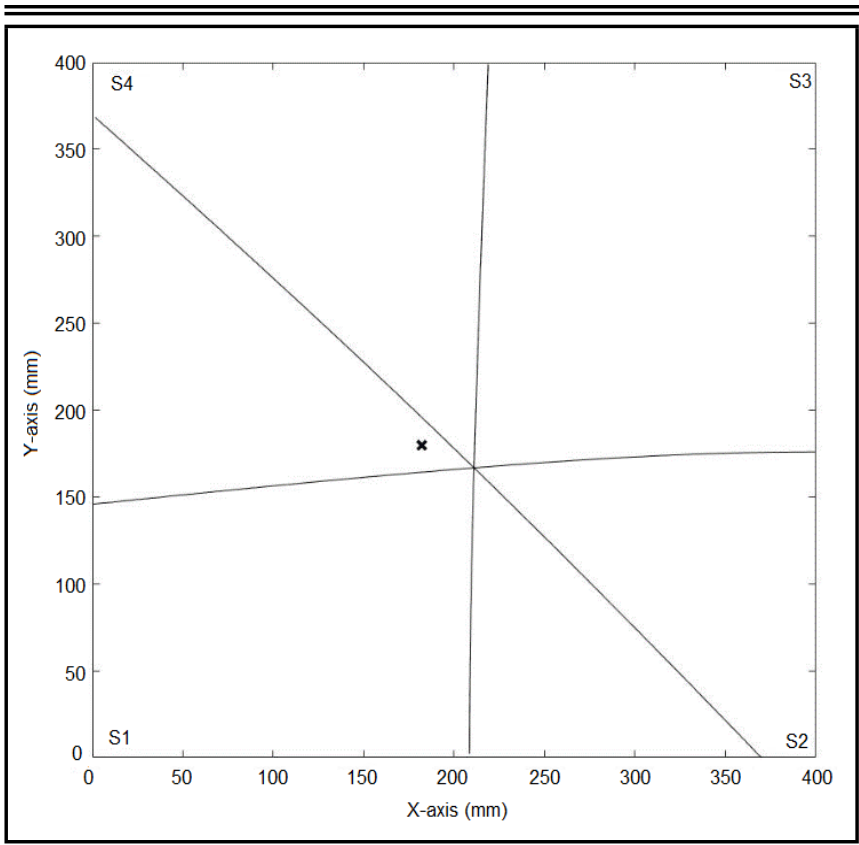

Figure 22. $T_{4}$ acting as an actuator.

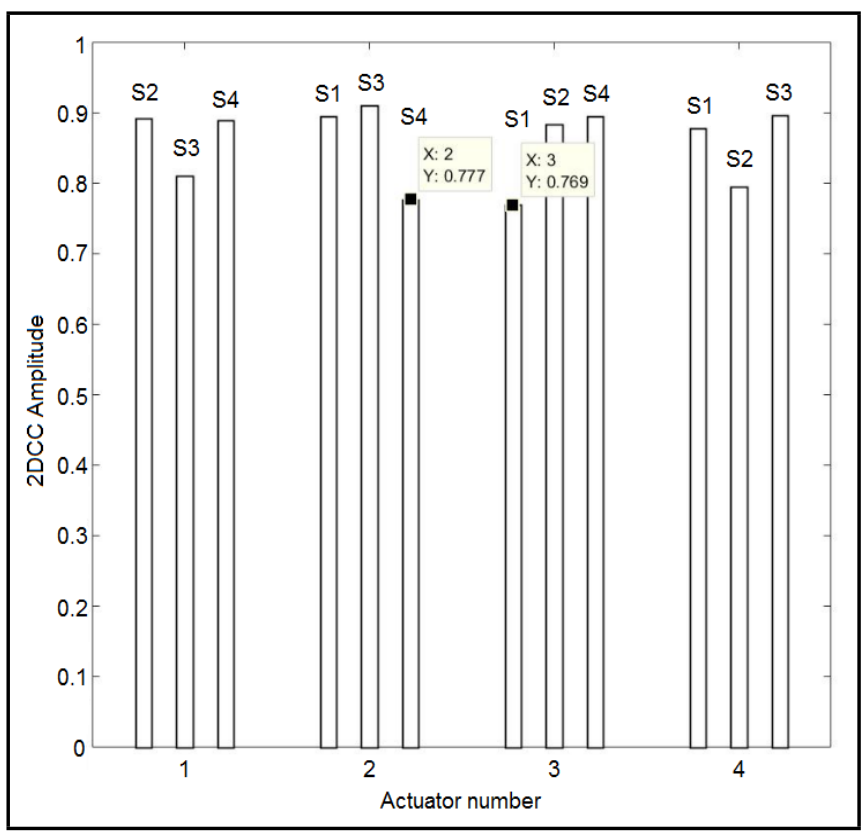

Figure 23. 2-D correlation coefficient.

of a faulty rivet in a riveted structure. The specimen and experimental setup used for this study are described in the following section.

\section{LOCALIZATION OF A DAMAGED RIVET IN A RIVETED STRUCTURE}

The specimen required for the study is fabricated by riveting a $40 \times 30$ sq. $\mathrm{cm}$ rectangular plate with a $30 \times 5$ sq. $\mathrm{cm}$ piece of a plate with Al flat-head rivets, as shown in Fig. 31. Four such specimens, with two of them riveted with intact rivets and two having one faulty rivet, as highlighted by the black colour in Fig. 32 (c) and (d), respectively, are fabricated. The rivet is damaged by cutting about $50 \%$ of its shank along the diameter direction, as shown schematically in Fig. 31. Figures 31 and 32 (a)-(d) show the front and rear views of the specimen, re-

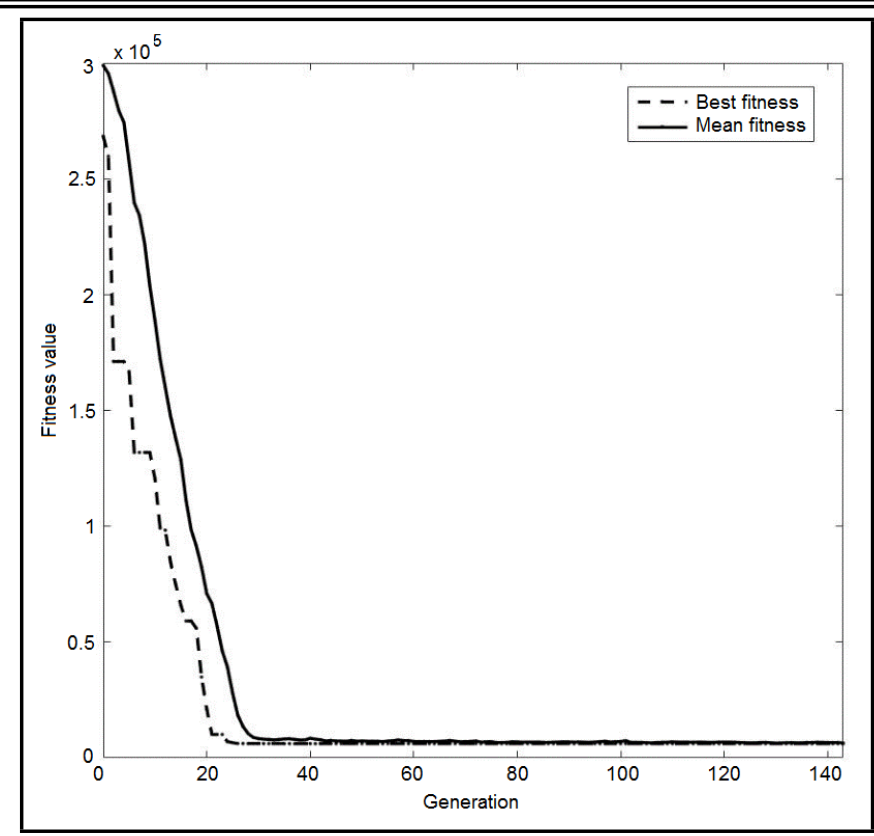

Figure 24. Convergence of fitness function.

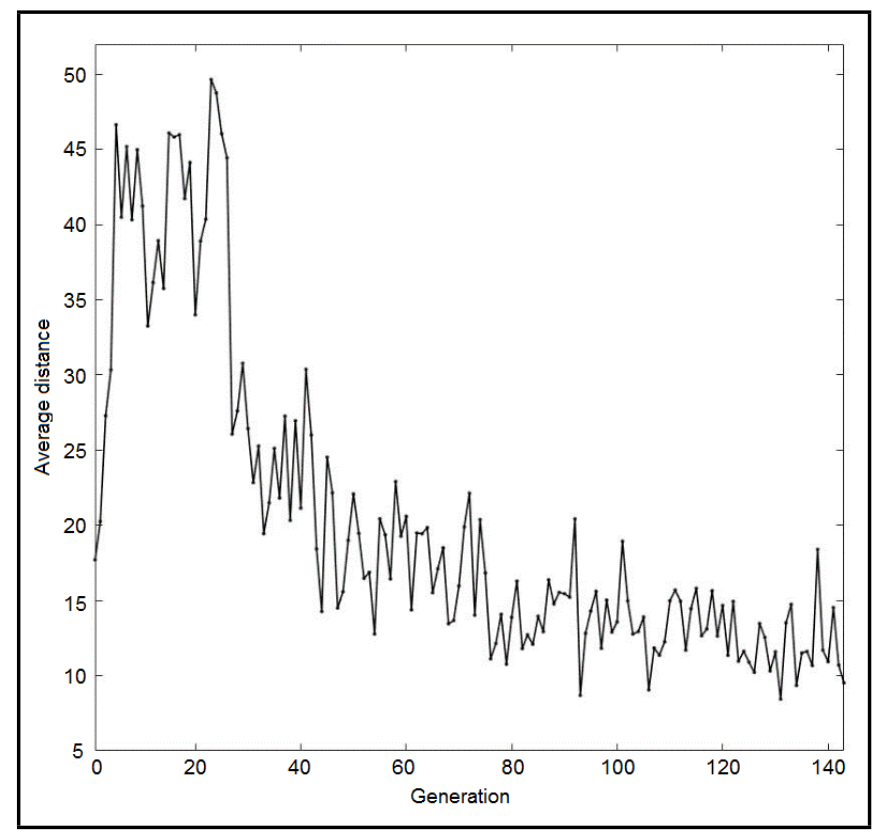

Figure 25. Distance between individuals.

spectively. Eight PW transducers are bonded onto the rear side of the specimens, as shown in Fig. 32. The PW transducers are used to actuate and sense the guided waves. The centre-tocentre distance between the PW transducers along the length of the larger side of the plate is $7.5 \mathrm{~cm}$.

The experimental setup used is similar to that shown in Fig. 3. An amplifier is used additionally in this case to amplify the signal strength by a factor of 50. A tone burst of $300 \mathrm{Vpp}$ sine wave modulated by Gaussian function is generated, using the function generator and amplifier, and it is given to the PW transducers for exciting the guided waves. These guided waves, after interrogating the specimen, are received by the oscilloscope and are further sent to the computer for offline signal processing. The experiments are carried out at a central frequency of $204 \mathrm{kHz}$. The wave responses are recorded for all possible actuator-sensor pairs across the rivets. 


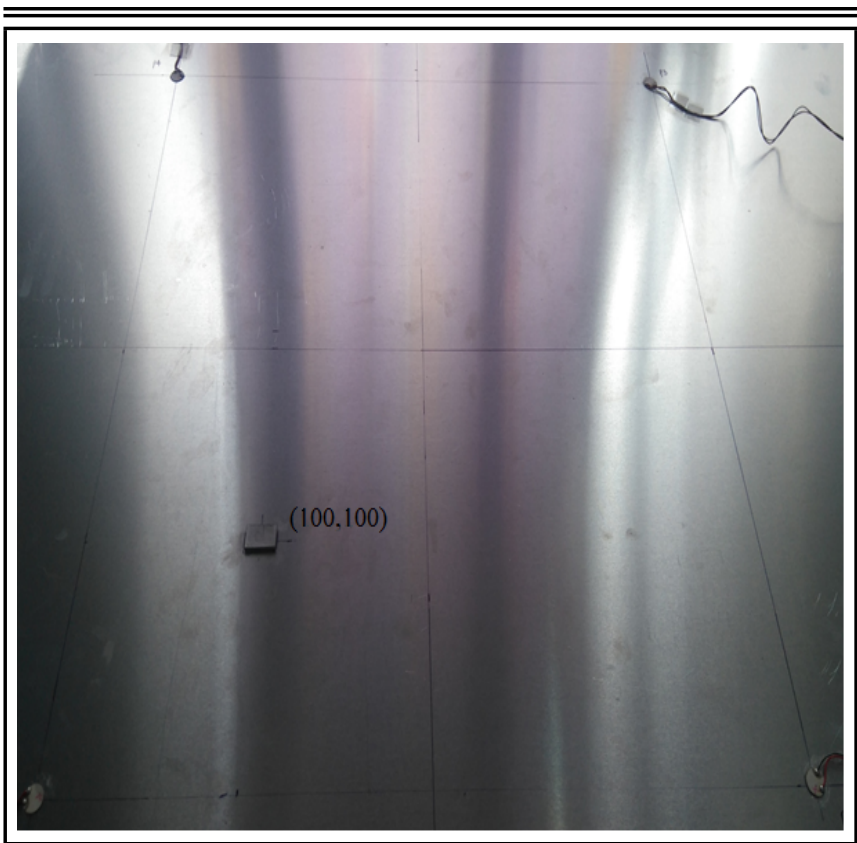

Figure 26. An extra material added onto the plate.

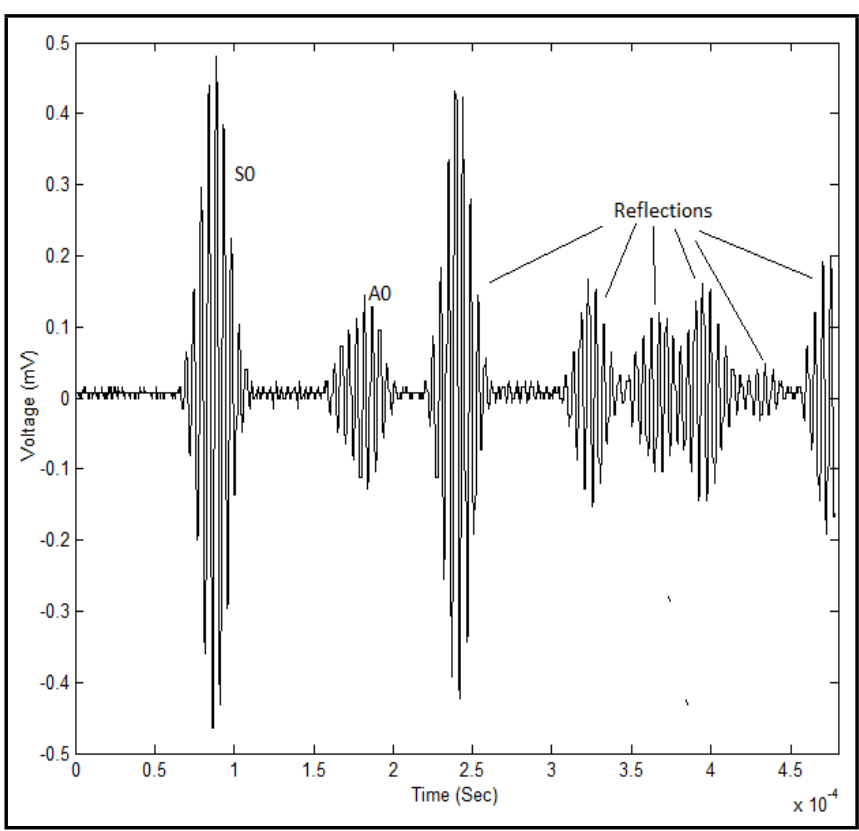

Figure 27. Healthy response for $T_{1} T_{2}$ configuration.

\subsection{Methodology for Locating the Damaged Rivet}

It is known that a closed crack in the path of guided waves produces higher harmonics in the waves. This phenomenon is attributed to the contact nonlinearity. To ensure the presence of higher harmonics in the wave response because of a crack in the rivet, the time domain data obtained in the experiments are converted into the frequency domain. The amplitudes of the second harmonics obtained in the cases of undamaged and damaged specimens are shown in Figs. 33-36 for all the actuatorsensor pairs. The undamaged specimens also show the presence of the second harmonics. These higher harmonics are because of the contact between two riveted plates, which also contribute to the higher harmonics observed in the damaged specimens. However, the presence of a crack in the rivet increases amplitudes of the second harmonics in the damaged specimens,

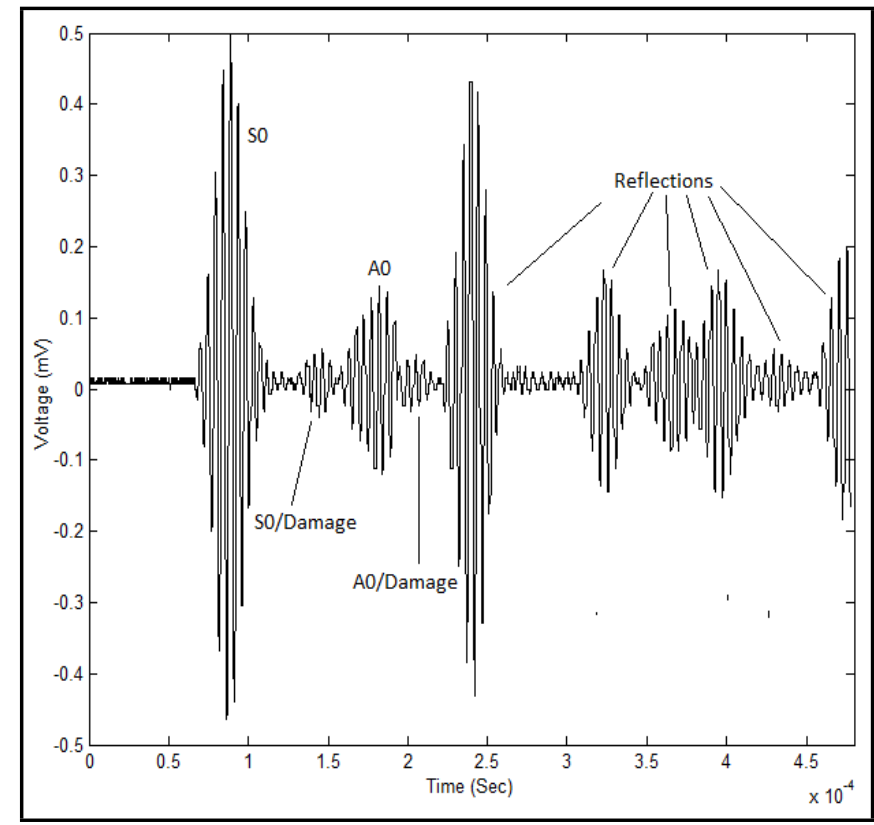

Figure 28. Damaged response for $T_{1} T_{2}$ configuration.

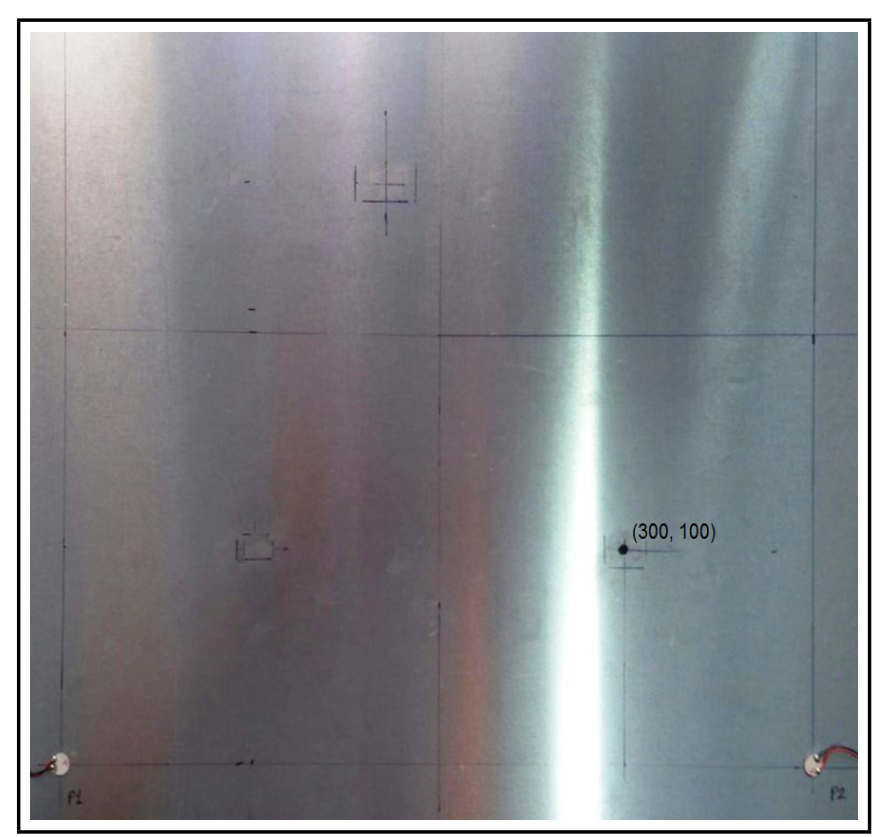

Figure 29. A through thickness hole in the plate.

which is evident form Figs. 33-36.

It is expected that the second harmonics will be of higher amplitudes in the cases of actuator-sensor paths across the cracked rivet. With this presumption, an algorithm is devised to locate the cracked rivet and it is explained here. The wave responses in the cases of healthy and damaged specimens are obtained first for all possible actuator-sensor paths across the rivets. Then, the two-dimensional CCs are computed using Eq. (10) for the data obtained from the healthy and damaged specimens for each actuator-sensor path respectively. The value of the $\mathrm{CC}$ indicates the degree of similarity between the two responses. The damage index (DI) is defined as DI $=1-$ CC. In this case, higher the DI, the higher is the possibility of closeness of damage to the actuator-sensor path. This is confined by using normal distribution function (Gaussian) as: 


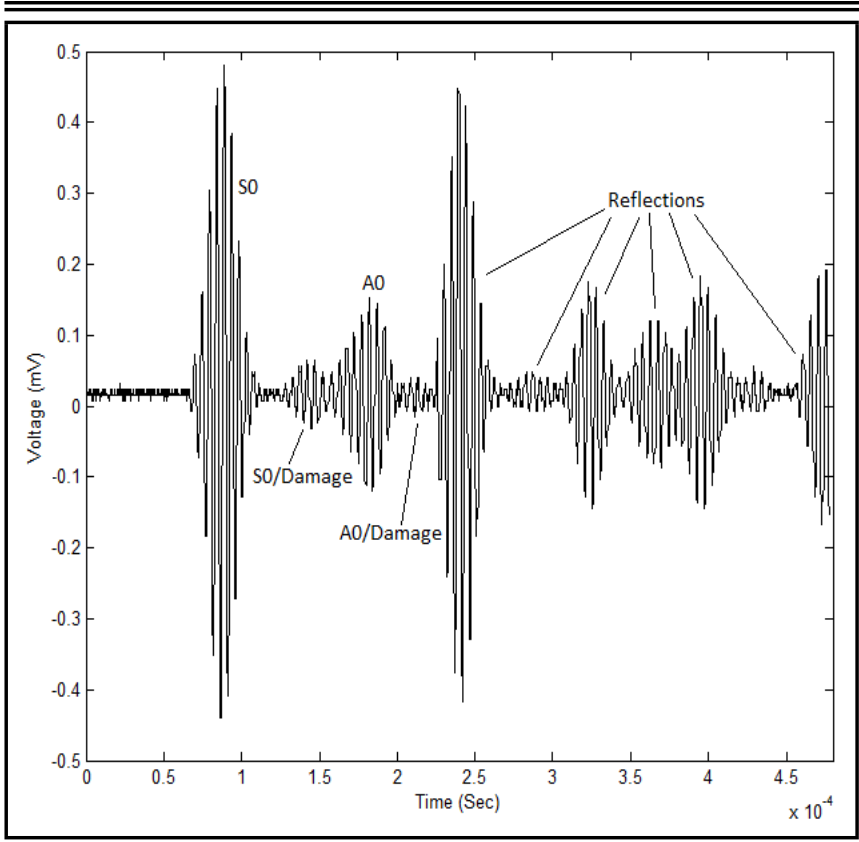

Figure 30. Damage response for $T_{1} T_{2}$ configuration.

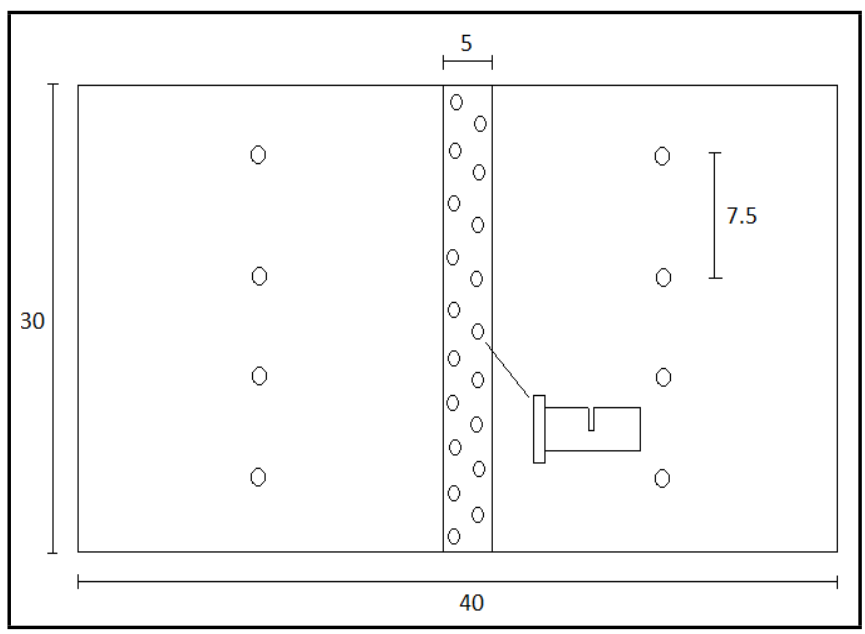

Figure 31. Schematic representation of the front view of the specimen (All dimensions are in $\mathrm{cm}$ ).

$$
\check{f}(\tilde{d})=\frac{1}{\sigma \sqrt{2 \pi}} e^{\frac{-(\tilde{d}-\mu)^{2}}{2 \sigma^{2}}} \text { for } \quad-\infty<\tilde{d}<+\infty .
$$

This means that the effect of the DI is maximum if the damage lies on the sensor-actuator path and it decreases gradually as the distance of the damage from the actuator-sensor path increases. Here, the value of $\tilde{d}$ is determined in an elliptical zone for each actuator-sensor path as:

$$
\tilde{d}=\frac{D_{a}+D_{s}}{D_{k}}-1
$$

where $D_{k}$ is the distance between the actuator and the sensor for the $k$ th sensing path and $D_{a}$ and $D_{s}$ are the distances from a pixel point to the actuator and sensor, respectively. In the present study, the values of the mean $\mu$ and the standard deviation $\sigma$ are set to be 0 and 40. The final result is found to be invariant with the change in these values. However, they are the measure of control of the size of ellipse. Assuming that there are $k$ actuator-sensor paths for damage identification in

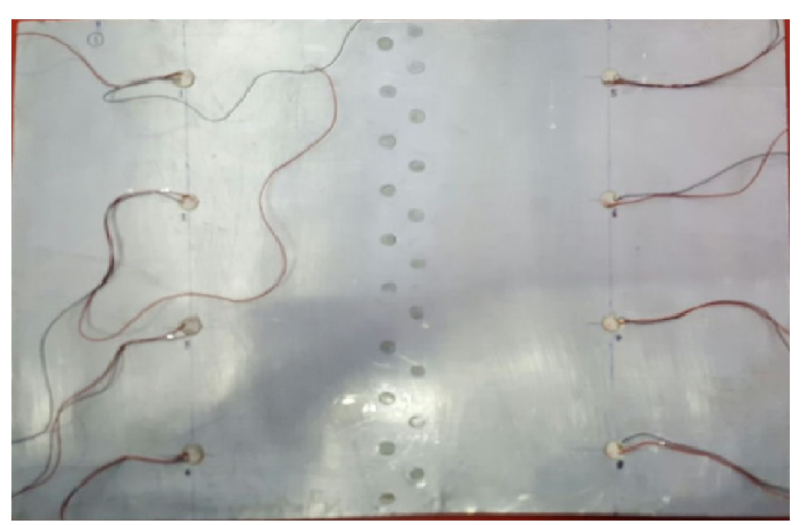

(a)

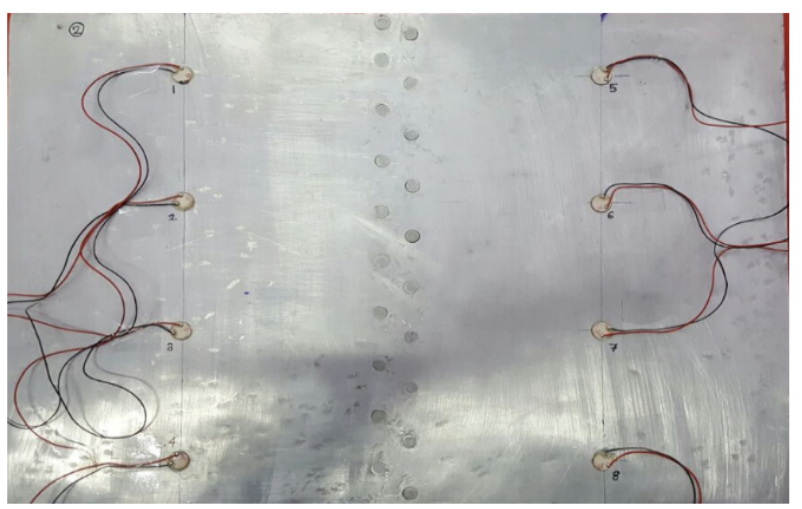

(b)

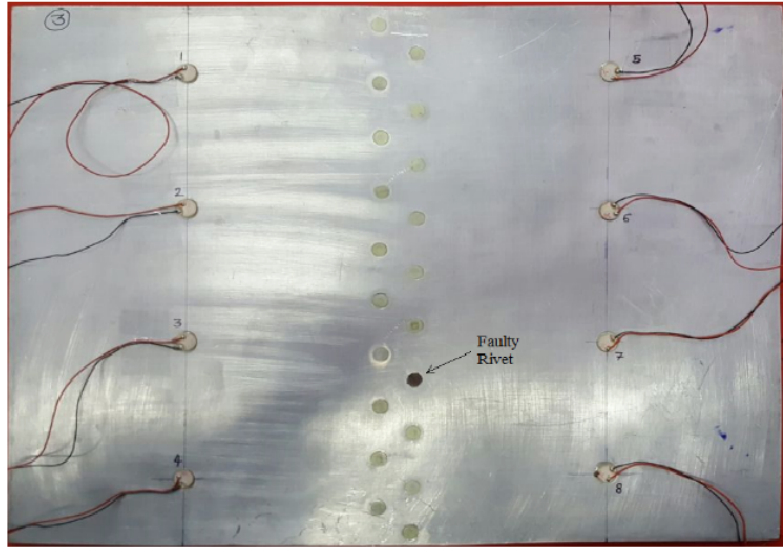

(c)

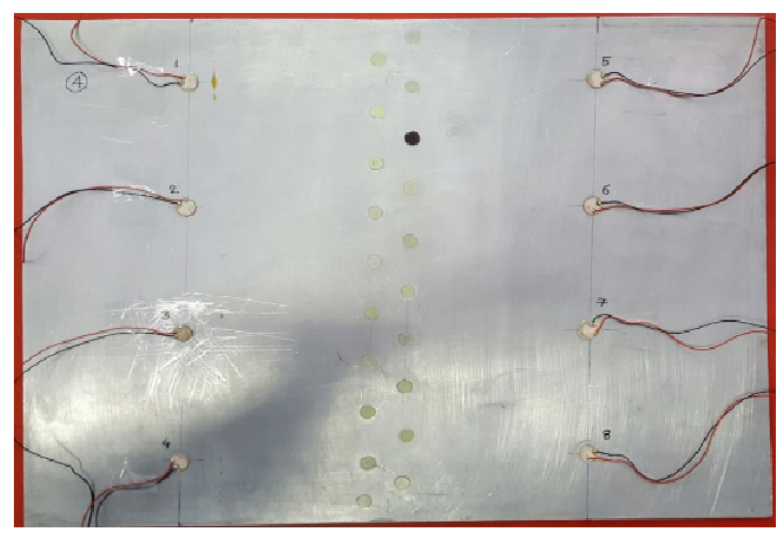

(d)

Figure 32. Rear view of the specimen (a) Undamaged specimen 1 (b) Undamaged specimen 2 (c) Damaged specimen 3 (d) Damaged specimen 4. 


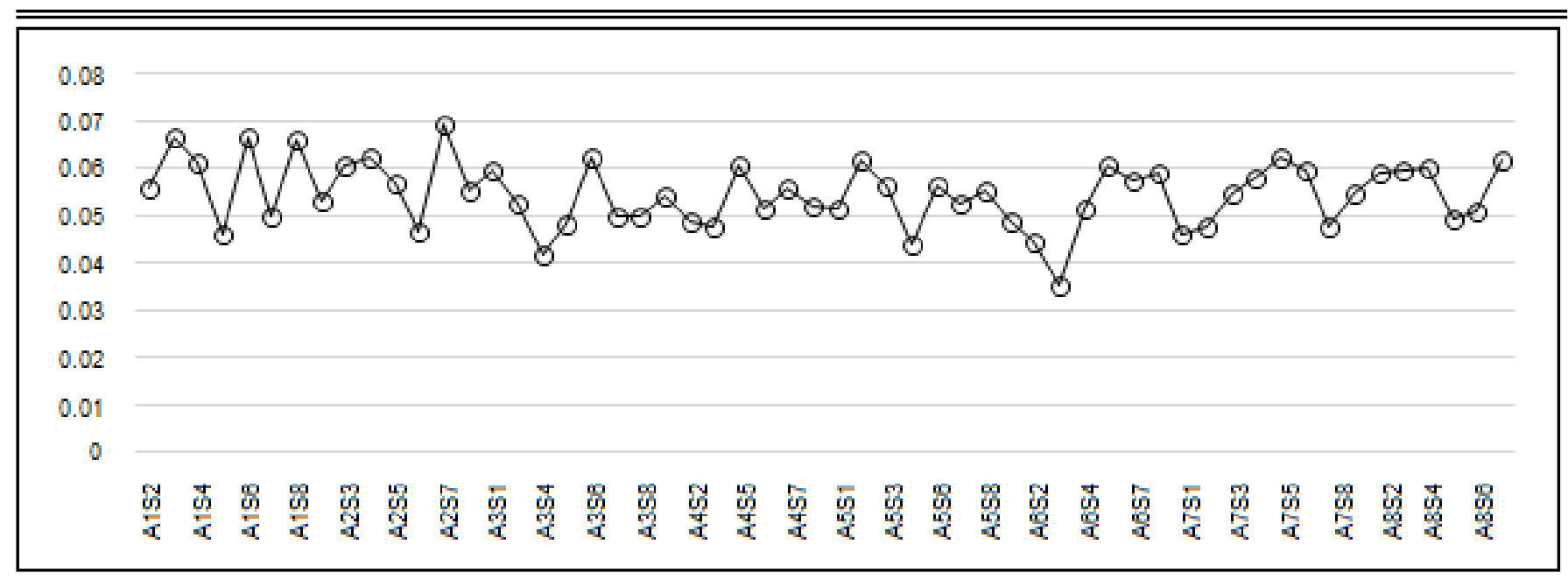

Figure 33. Amplitudes of the second harmonics in case of undamaged specimen 1 (FFT amplitude on ordinate and all actuator-sensor configurations on abscissa).

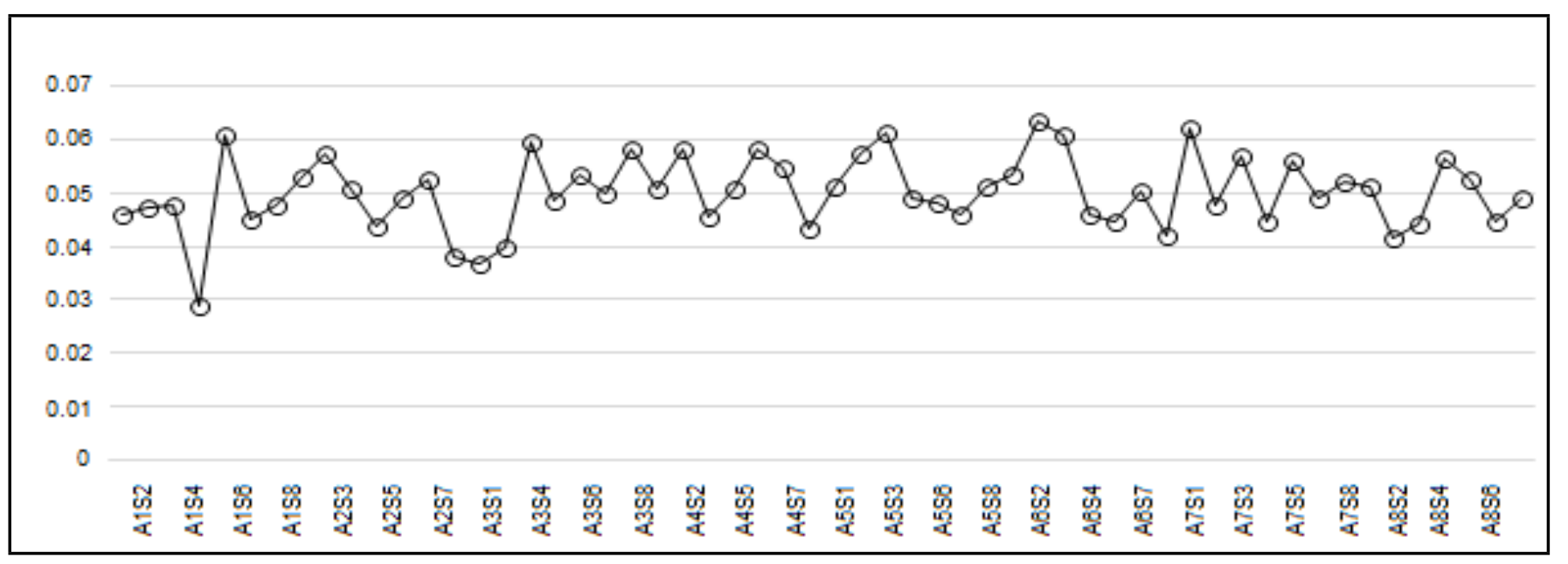

Figure 34. Amplitudes of the second harmonics in case of undamaged specimen 2 (FFT amplitude on ordinate and all actuator-sensor configurations on abscissa).

the transducer network, the probability of the presence of damage at a pixel position $(x, y)$ can be written as:

$$
P(x, y)=\sum_{i=1}^{k} D I_{k} \check{f}(\tilde{d})_{k} A(2 \oint)_{k} ;
$$

where $P$ is the pixel value, $D I_{k}$ is the damage index for the $k$ th path, $\check{f}(\tilde{d})_{k}$ is the normal distribution function, and $A(2 \oint)_{k}$ is the amplitude of the second harmonic. An increase in the magnitude of $A(2 £)_{k}$ is seen in the actuator-sensor path close to the damage. Thus, $A(2 £)_{k}$ in Eq. (16) influences the probability of presence of damage in a given actuator-sensor path to a greater extent. The results obtained are shown in Figs. 37 and 38, which show the location of damaged rivet in the specimens. Two cases are considered in the present study in order to test the genericness of the devised algorithm. The output is not showing the exact location of the damaged rivet in both the cases. This can be attributed to the fact that the contact area of the crack in a rivet is too small, as compared to the contact area of the riveted plates in the specimens.

\section{CONCLUSIONS}

In the present work, new algorithms are proposed to localize different types of damages in the plain and riveted $\mathrm{Al}$ specimens. The results obtained in the case of a plain Al specimen show that the proposed method can locate the damage within an overall error of less than $2 \%$. The error is measured with respect to the maximum of deviation of either coordinates from the respective actual damage coordinates. The error is reasonably small because the damage localization is done in two steps. However, the accuracy of the localization can be further increased by increasing the number of transducers and intersection points. The objective function can then be formulated accordingly with appropriate weighted values. The two-dimensional correlation coefficient analysis, in this case, may give more detailed information about the location of the damage, with respect to the transducer positions. The results obtained in the case of riveted specimens confirm that the method and algorithm seem to have great potential for locating the faulty rivets, as it combines the linear as well as nonlinear approaches. Exact faulty rivet has not been localized in the present study, but the location predicted is close to the actual location of the faulty rivet. The error can be attributed to the inconsistent manufacturing qualities of the in-house fabricated damaged and undamaged specimens and contact nonlinearity between the plates itself. Still, the method could broadly give the location of the faulty rivet. The proposed algorithm may be beneficial to identify a small area out of the bigger surface area of an aircraft with a huge number of rivets, wherein the faulty rivet lies. In this small area, some other techniques can be used further to identify the exact location of the faulty rivet or all the rivets in the identified area can be replaced as neighbouring 


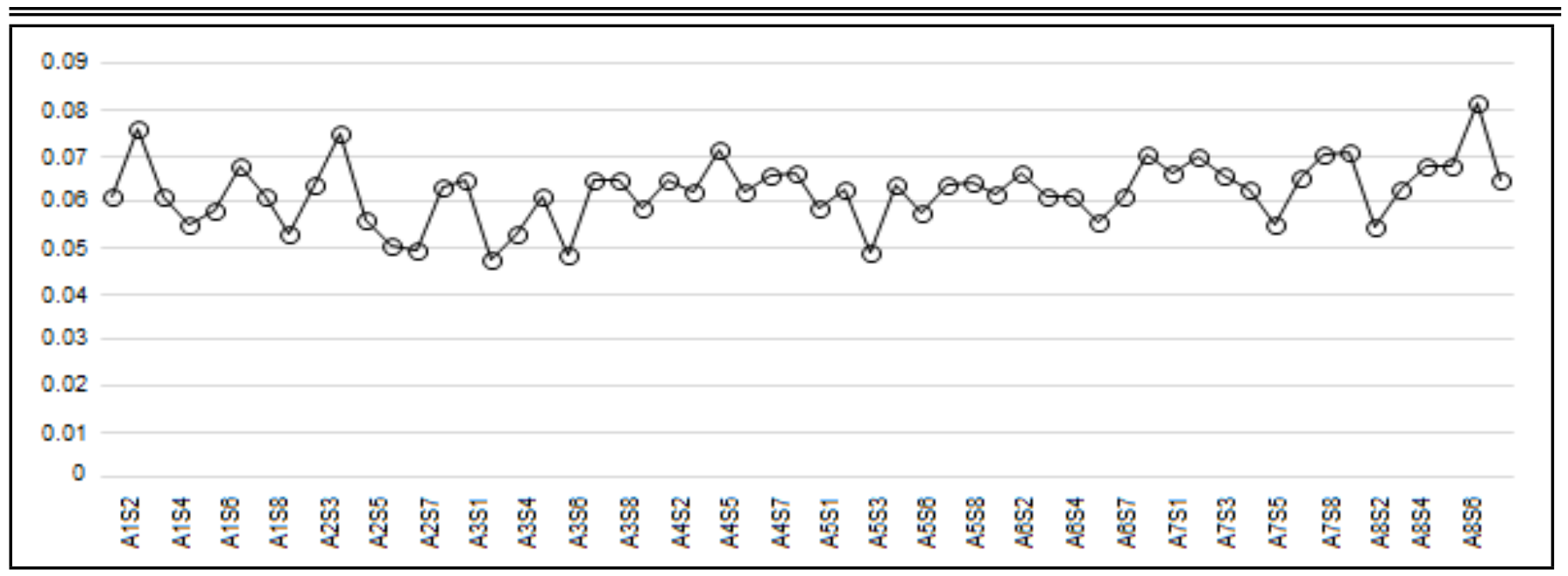

Figure 35. Amplitudes of the second harmonics in case of damaged specimen 3 (FFT amplitude on ordinate and all actuator-sensor configurations on abscissa).

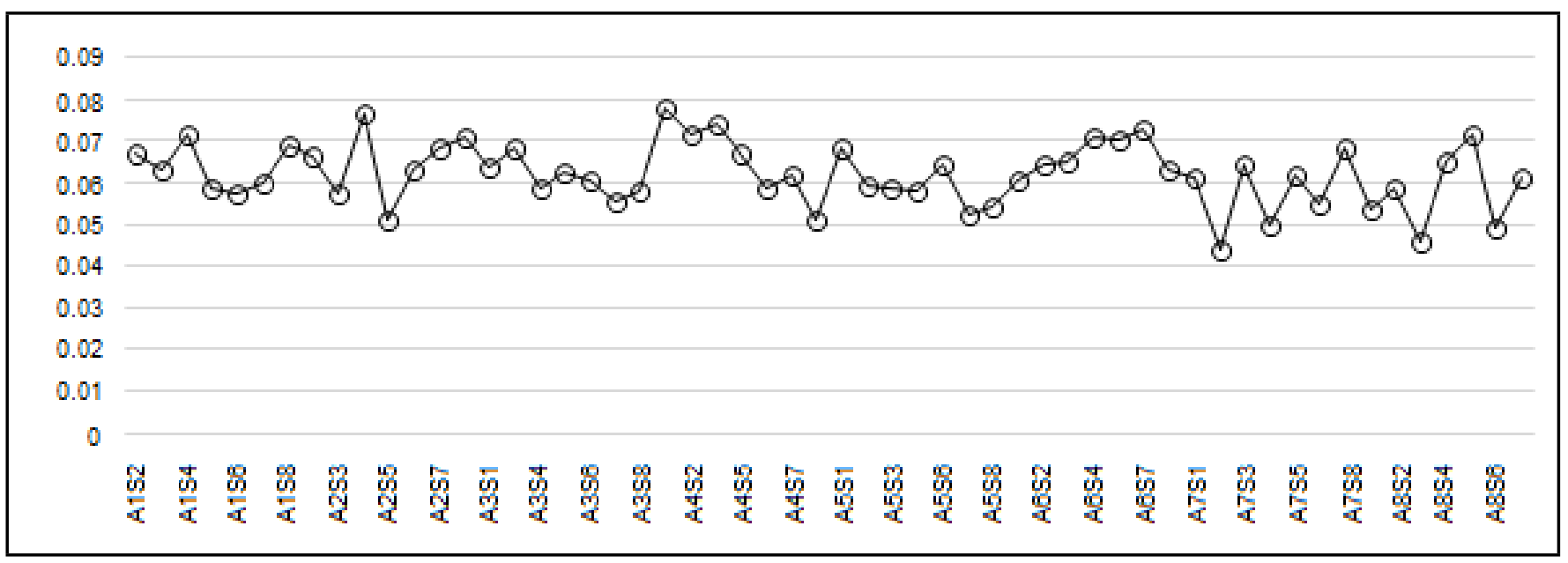

Figure 36. Amplitudes of the second harmonics in case of damaged specimen 4 (FFT amplitude on ordinate and all actuator-sensor configurations on abscissa).

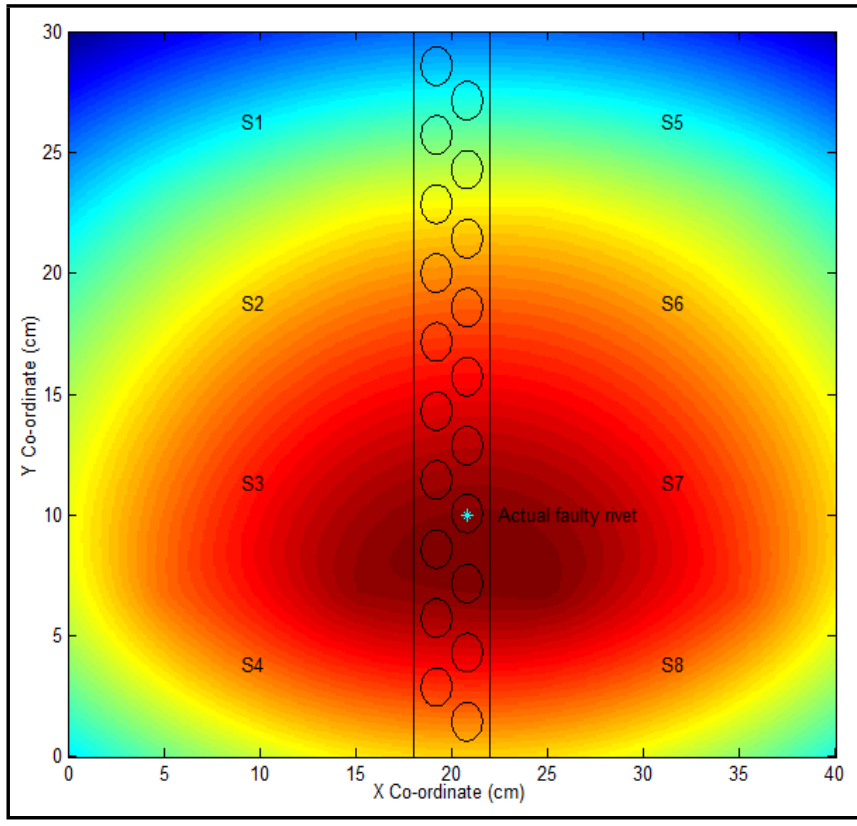

Figure 37. Damaged rivet location in specimen 3.

rivets may also be following the failure mechanism of the failed rivet.

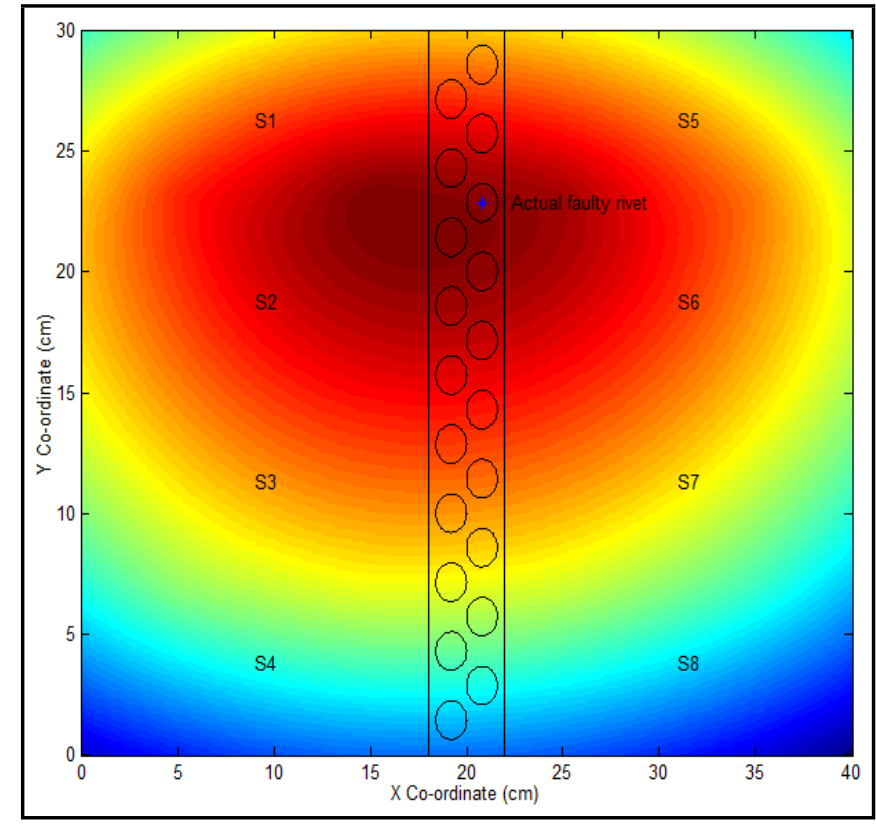

Figure 38. Damaged rivet location in specimen 4.

\section{REFERENCES}

1 Raghavan, A. and Cesnik, C. E. S. Review of guided-wave structural health monitoring, Shock 
and Vibration Digest, 39 (2), 91-114, (2007). https://dx.doi.org/10.1177/0583102406075428

2 Giurgiutiu, V., Bao, J., and Zhao, W. Piezoelectric wafer active sensor embedded ultrasonics in beams and plates, Experimental Mechanics, 43 (4), 428-449, (2003). https://dx.doi.org/10.1007/BF02411348

3 Su, Z., Ye, L., and Lu, Y. Guided Lamb waves for identification of damage in composite structures: A review, Journal of Sound and Vibration, 295 (3-5), 753-780, (2006). https://dx.doi.org/doi.org/10.1016/j.jsv.2006.01.020

${ }^{4} \mathrm{Su}, \mathrm{Z}$., and Ye, L. Fundamental Lamb mode-based delamination detection for CF/EP composite laminates using distributed piezoelectrics, Structural Health Monitoring, 3 (1), 43-68, (2004). https://dx.doi.org/10.1016/j.jsv.2006.01.020

5 Lemistre, M., Gouyon, R., Kaczmarek, H., and Balageas, D. Damage localization in composite plates using wavelet transform processing on Lamb wave signals, In: Chang, F. K. (ed.), Structural Health Monitoring, 2nd ed., Lancaster: Technomic, 861-870, (1999).

${ }^{6}$ Masurkar, F. A., Yelve, N. P., and Mitra, M. Lamb wave based experimental and FE simulation studies for damage detection in metallic and composite plates using Geodesic algorithm, Proceedings of 7th ISSS International Conference on Smart Materials, Structures, and Systems, Bangalore, India, (2014).

${ }^{7}$ Masurkar, F. A., and Yelve, N. P. Locating a damage in an aluminium plate using Lamb waves, Proceedings of 25th National Seminar and International Exhibition on NDE, Hyderabad, India, (2015).

8 Gangadharan, R., Bhat, M. R., Murthy, C. R. L., and Gopalakrishnan, S. A geodesic-based triangulation technique for damage location in metallic and composite plates, Smart Material and Structures, 19 (11), (2010). https://dx.doi.org/10.1088/0964-1726/19/11/115010

9 Hamstad, M. A., O'Gallagher, A., and Gary, J. A Wavelet transform applied to acoustic emission signals: Part 2: Source location, Journal of Acoustic Emission, 20, 62-82, (2002).

10 Ambrozinski, L., Packo, P., Stepinski, T., and Uhl, T. Ultrasonic guided waves based method for SHM simulations and an Experimental test, Proceedings of 5th World Conference on Structural Control and Monitoring, Tokyo, Japan, (2010).

11 Liu, Y., Yekani Fard, M., Chattopadhyay, A., and Doyle, D., Damage assessment of CFRP composites using a time-frequency approach, Journal of Intelligent Material Systems and Structures, 23 (4), 397-413, (2012). https://dx.doi.org/10.1177/1045389X11434171

12 Wang, C. S., and Chang, F. K. Built-in diagnostics for impact damage identification of composite structures, In: Chang, F. K. (ed.), Structural Health Monitoring, 2nd ed. Lancaster: Technomic, 612-621, (1999).
13 Ostachowicz, W., Kudela, P., Malinowski, P., and Wandowski, T. Damage localization in plate-like structures based on PZT sensors, Mechanical Systems and Signal Processing, 23 (6), 1805-1829, (2009). https://dx.doi.org/10.1016/j.ymssp.2008.10.011

14 Liu, X., Zhou, C., and Jiang, Z. Damage localization in plate-like structure using built-in PZT sensor network, Smart Structures and Systems, 9 (1), 21-33, (2012). https://dx.doi.org/10.1.1.668.4123

15 Yan, G., Zhou, L. L., and Yuan, F. G. Wavelet-based built-in damage detection and identification for composites, Proceedings of the SPIE, 5765, 324-334, (2005). https://dx.doi.org/doi.org/10.1117/12.602435

16 Sharif-Khodaei, Z., and Aliabadi, M. H. Assessment of delay-and-sum algorithms for damage detection in aluminium and composite plates, Smart Materials and Structures, 23 (7), 1-20, (2014). https://dx.doi.org/10.1088/0964-1726/23/7/075007

17 Ostachowicz, W., and Radzienski, M. Structural health monitoring by means of elastic wave propagation, Journal of Physics, Conference Series, 382 (1), 1-8, (2003). https://dx.doi.org/10.1088/1742-6596/382/1/012003

18 Wang, D., He, J., Dong, B., Liu, X., and Zhang, W. Novel damage detection techniques for structural health monitoring using a hybrid sensor, Mathematical Problems in Engineering, 2016 (1), 1-13, (2016). https://dx.doi.org/10.1155/2016/3734258

19 Masurkar, F. A., and Yelve, N. P. Optimizing location of damage within an enclosed area defined by an algorithm based on the Lamb wave response data, Applied Acoustics, 120, 98-110, (2017). https://dx.doi.org/10.1016/j.apacoust.2017.01.014

20 Rose, J. L., Ultrasonic waves in Solid media, Cambridge University Press, (1999).

21 Liu, X., Leimbach, K. R., Hartmann, D., and Hoffer, R. Signal analysis using wavelets for structural damage detection applied to wind energy converters, Proceedings of 14th International conference on Computing in Civil and Building Engineering, Moscow, Russia, (2012).

22 Ip, K. H., Tse, P. W., and Tam, H. Y. Extraction of patch-induced Lamb waves using a wavelet transform, Smart Materials and Structures, 13 (4), 861-872, (2004). https://dx.doi.org/10.1088/0964-1726/13/4/024 